\title{
Traveling Pulses and Wave Propagation Failure in Inhomogeneous Neural Media*
}

\author{
Zachary P. Kilpatrick ${ }^{\dagger}$, Stefanos E. Folias ${ }^{\ddagger}$, and Paul C. Bressloff ${ }^{\dagger}$
}

\begin{abstract}
We use averaging and homogenization theory to study the propagation of traveling pulses in an inhomogeneous excitable neural network. The network is modeled in terms of a nonlocal integrodifferential equation, in which the integral kernel represents the spatial distribution of synaptic weights. We show how a spatially periodic modulation of homogeneous synaptic connections leads to an effective reduction in the speed of a traveling pulse. In the case of large amplitude modulations, the traveling pulse represents the envelope of a multibump solution, in which individual bumps are nonpropagating and transient. The appearance (disappearance) of bumps at the leading (trailing) edge of the pulse generates the coherent propagation of the pulse. Wave propagation failure occurs when activity is insufficient to maintain bumps at the leading edge.
\end{abstract}

Key words. traveling waves, excitatory neural network, inhomogeneous media, homogenization, neural field theory, wave propagation failure

AMS subject classification. $92 \mathrm{C} 20$

DOI. $10.1137 / 070699214$

1. Introduction. Traveling waves of electrical activity have been observed in vivo in a number of sensory cortical areas including the somatosensory cortex of behaving rats [30], turtle and mollusk olfactory bulbs [22, 23], turtle cortex [34], and visuomotor cortices in the cat [36]. Such waves are usually seen during periods without sensory stimulation; the subsequent presentation of a stimulus then induces a switch to synchronous oscillatory behavior [13]. Traveling waves are also a characteristic feature of certain neurological disorders in humans, including epilepsy [8] and migraines [24]. Therefore, investigating the mechanisms underlying wave propagation in neural tissue is important for understanding both normal and pathological brain states. A common experimental paradigm is to record electrical activity in vitro using thin slices of cortical tissue, in which inhibition has been suppressed by blocking $\mathrm{GABA}_{A}$ receptors with an antagonist such as bicuculline $[7,15,40,35,32,18]$. Synchronized discharges can then be evoked by a weak electrical stimulus from any site on the cortical slice. Following rapid vertical propagation, each discharge propagates away from the stimulus in both horizontal directions at a mean velocity of about $6-9 \mathrm{~cm} / \mathrm{s}$. Although the conditions for wave propagation may differ from the intact cortex due to the removal of some long-range connections during slice preparation, the in vitro slice is more amenable to pharmacological manipulation and to multielectrode recordings.

\footnotetext{
${ }^{*}$ Received by the editors August 3, 2007; accepted for publication (in revised form) by B. Ermentrout October 5 , 2007; published electronically January 23, 2008. This research was supported by NSF grants DMS-0515725 and IGERT-0217424.

http://www.siam.org/journals/siads/7-1/69921.html

${ }^{\dagger}$ Department of Mathematics, University of Utah, 155 S 1400 E, Salt Lake City, UT 84112 (kilpatri@math.utah. edu, bressloff@math.utah.edu).

${ }^{\ddagger}$ Department of Mathematics and Statistics, Boston University, 111 Cummington Street, Boston, MA 02215 (sf@ math.bu.edu).
} 
Mathematical analyses of cortical wave propagation typically consider reduced one-dimensional network models. Under the additional assumption that the synaptic interactions are homogeneous, it has been shown that an excitatory neural network supports the propagation of a traveling front $[12,19,5]$ or, in the presence of slow adaptation, a traveling pulse $[39,1,31,42,43,10,11,14,38]$. However, the patchy nature of long-range horizontal connections in superficial layers of certain cortical areas suggests that the cortex is more realistically modeled as an inhomogeneous neural medium. For example, in the primary visual cortex the horizontal connections tend to link cells with similar stimulus feature preferences such as orientation and ocular dominance [28, 41, 2]. Moreover, these patchy connections tend to be anisotropic, with the direction of anisotropy correlated with the underlying orientation preference map. Hence the anisotropic pattern of connections rotates approximately periodically across the cortex resulting in a periodic inhomogeneous medium [3, 4]. Another example of inhomogeneous horizontal connections is found in the prefrontal cortex [27, 29, 17], where pyramidal cells are segregated into stripes that are mutually connected via horizontally projecting axon collaterals; neurons within the gaps between stripes do not have such projections.

In this paper we investigate how a spatially periodic modulation of long-range synaptic weights affects the propagation of traveling pulses in a one-dimensional excitatory neural network, extending previous work on traveling fronts in neural network models [3] and reactiondiffusion systems [20,21]. We proceed by introducing a slowly varying phase into the traveling wave solution of the unperturbed homogeneous network, and then we use perturbation theory to derive a dynamical equation for the phase, from which the mean speed of the wave can be calculated. We show that a periodic modulation of the long-range connections slows down the wave, and if the amplitude and wavelength of the periodic modulation is sufficiently large, then wave propagation failure can occur. A particularly interesting result of our analysis is that in the case of large amplitude modulations, the traveling pulse is no longer superthreshold everywhere within its interior, even though it still propagates as a coherent solitary wave. We find that the pulse now corresponds to the envelope of a multibump solution, in which individual bumps are nonpropagating and transient. The appearance (disappearance) of bumps at the leading (trailing) edge of the pulse generates the propagation of activity; propagation failure occurs when activity is insufficient to create new bumps at the leading edge.

2. Inhomogeneous network model. Consider a one-dimensional neural network model of the form [31]

$$
\begin{aligned}
\tau_{m} \frac{\partial u(x, t)}{\partial t} & =-u(x, t)+\int_{-\infty}^{\infty} w\left(x, x^{\prime}\right) f\left(u\left(x^{\prime}, t\right)\right) d x^{\prime}-\beta v(x, t) \\
\frac{1}{\alpha} \frac{\partial v(x, t)}{\partial t} & =-v(x, t)+u(x, t)
\end{aligned}
$$

where $u(x, t)$ is the population activity at position $x \in \mathbb{R}, \tau_{m}$ is a membrane time constant, $f(u)$ is the output firing rate function, $w\left(x, x^{\prime}\right)$ is the excitatory connection strength from neurons at $x^{\prime}$ to neurons at $x$, and $v(x, t)$ is a local negative feedback mechanism, with $\beta$ and $\alpha$ determining the relative strength and rate of feedback. This type of feedback, which could be spike frequency adaptation or synaptic depression, favors traveling waves [1, 31]. The nonlinearity $f$ is a smooth monotonic increasing function, 


$$
f(u)=\frac{1}{1+e^{-\eta(u-\kappa)}},
$$

where $\eta$ is a gain parameter and $\kappa$ is a threshold. As $\eta \rightarrow \infty, f \rightarrow H$, where $H(u)=\Theta(u-\kappa)$ and

$$
\Theta[u]= \begin{cases}0, & u \leq 0 \\ 1, & u>0 .\end{cases}
$$

The periodic microstructure of the cortex is incorporated by taking the weight distribution to be of the form $[3,4]$

$$
w\left(x, x^{\prime}\right)=W\left(\left|x-x^{\prime}\right|\right)\left[1+\mathcal{D}^{\prime}\left(\frac{x^{\prime}}{\varepsilon}\right)\right],
$$

where $\mathcal{D}$ is a $2 \pi$-periodic function and $\varepsilon$ determines the microscopic length-scale. (We consider the first-order derivative of $\mathcal{D}$ so that the zeroth-order harmonic is explicitly excluded.) It is important to note that (2.4) is a one-dimensional abstraction of the detailed anatomical structure found in the two-dimensional layers of real cortex. (See [6] for a more detailed discussion of cortical models.) However, it captures both the periodic-like nature of longrange connections and possible inhomogeneities arising from the fact that this periodicity is correlated with a fixed set of cortical feature maps.

For concreteness, we take the homogeneous weight function $W$ to be an exponential,

$$
W(x)=\frac{W_{0}}{2 d} \mathrm{e}^{-|x| / d}
$$

where $d$ is the effective range of the excitatory weight distribution, and set

$$
\mathcal{D}(x)=\rho \sin (x), \quad 0 \leq \rho<1,
$$

where $\rho$ is the amplitude of the periodic modulation. We require that $0 \leq \rho<1$ so that the weight distribution remains nonnegative everywhere. Example plots of the resulting weight function $w(x, y)$ of (2.4) are shown in Figure 1 for fixed $x$. This illustrates both the periodic modulation and the associated network inhomogeneity, since the shape of the weight distribution varies periodically as the location $x$ of the postsynaptic neuron shifts. Plotting $w\left(x, x^{\prime}\right)$ for fixed $x^{\prime}$ simply gives an exponential distribution whose maximum depends on $x^{\prime}$. Finally, the temporal and spatial scales of the network are fixed by setting $\tau_{m}=1, d=1$, and the scale of the synaptic weights is fixed by setting $W_{0}=1$. The membrane time constant is typically around $10 \mathrm{~ms}$ and the length-scale of synaptic connections is typically $1 \mathrm{~mm}$. Thus, in dimensionless units the speed of an experimentally measured wave will be $c=\mathcal{O}(1)$.

3. Averaging theory and homogenization. Our goal in this paper is to determine how the periodic modulation of the weight function affects properties of traveling pulses in the one-dimensional system obtained by substituting (2.4) into (2.1):

$$
\begin{aligned}
\frac{\partial u(x, t)}{\partial t} & =-u(x, t)+\int_{-\infty}^{\infty} W\left(\left|x-x^{\prime}\right|\right)\left[1+\mathcal{D}^{\prime}\left(\frac{x^{\prime}}{\varepsilon}\right)\right] f\left(u\left(x^{\prime}, t\right)\right) d x^{\prime}-\beta v(x, t), \\
\frac{1}{\alpha} \frac{\partial v(x, t)}{\partial t} & =-v(x, t)+u(x, t) .
\end{aligned}
$$

Copyright $@$ by SIAM. Unauthorized reproduction of this article is prohibited. 

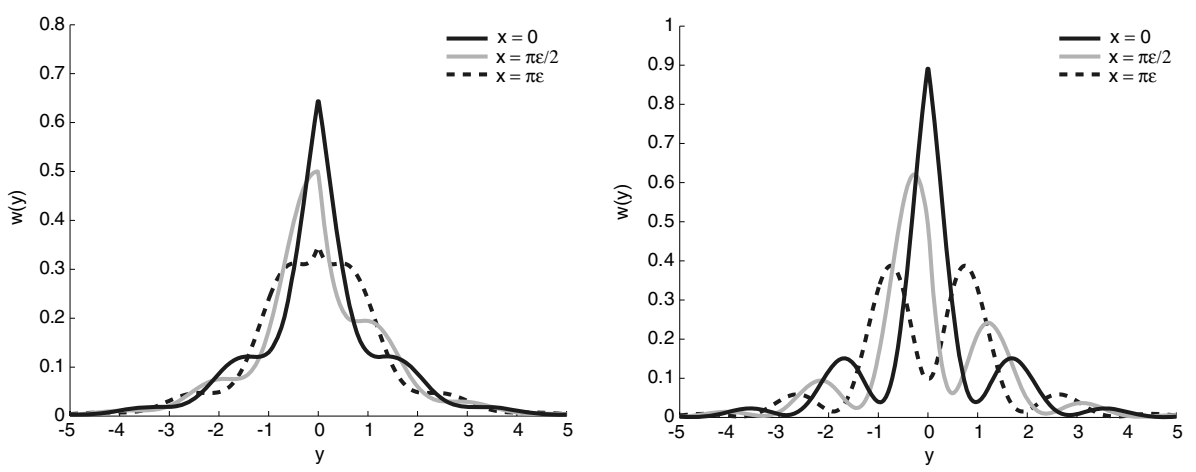

Figure 1. Left: Weight kernel $w(y)=w(x, y)$ for a neuron centered at $x=0, \pi \varepsilon / 2, \pi \varepsilon$ when $\rho=0.3$, and $\varepsilon=0.3$. Right: Corresponding weight kernel when $\rho=0.8$ and $\varepsilon=0.3$.

Assuming $\varepsilon$ is a small parameter (in units of the space constant $d$ ), a zeroth-order approximation to (3.1) can be generated by performing spatial averaging with respect to the periodic weight modulation, leading to the homogeneous system given by (2.1) with weight distribution $w\left(x, x^{\prime}\right)=W\left(\left|x-x^{\prime}\right|\right)$. Suppose that the homogeneous network supports the propagation of a traveling pulse of constant speed $c$. That is, $u(x, t)=U(\xi), v(x, t)=V(\xi)$, where $\xi=x-c t$ is the traveling wave coordinate, and $U(\xi), V(\xi) \rightarrow 0$ as $\xi \rightarrow \pm \infty$. Substituting such a solution into (2.1) with $w\left(x, x^{\prime}\right)=W\left(\left|x-x^{\prime}\right|\right)$ gives

$$
\begin{aligned}
-c U^{\prime}(\xi) & =-U(\xi)+\int_{-\infty}^{\infty} W\left(\xi-\xi^{\prime}\right) f\left(U\left(\xi^{\prime}\right)\right) d \xi^{\prime}-\beta V(\xi), \\
-\frac{c}{\alpha} V^{\prime}(\xi) & =-V(\xi)+U(\xi) .
\end{aligned}
$$

Assuming the existence of a solution $(U(\xi), V(\xi))$ to (3.2), we would like to determine whether or not a traveling wave persists in the presence of the periodic weight modulation. A crucial requirement for trajectories of the averaged homogeneous system to remain sufficiently close to trajectories of the exact inhomogeneous system for sufficiently small $\varepsilon$ is that solutions of the averaged system be structurally stable [16]. However, traveling pulses correspond to homoclinic trajectories within a dynamical systems framework and are thus not structurally stable. Therefore, one must go beyond lowest-order averaging to resolve differences between the homogeneous and inhomogeneous systems. We will proceed by carrying out a perturbation expansion in $\varepsilon$, extending previous work on traveling fronts in reaction-diffusion systems [20, 21] and excitable neural networks [3].

We begin by performing an integration by parts on the first equation in the system (3.1) so that

$$
\begin{aligned}
\frac{\partial u(x, t)}{\partial t}= & -u(x, t)+\int_{-\infty}^{\infty} W\left(x-x^{\prime}\right) f\left(u\left(x^{\prime}, t\right)\right) \mathrm{d} x^{\prime} \\
& +\varepsilon \int_{-\infty}^{\infty} \mathcal{D}\left(\frac{x^{\prime}}{\varepsilon}\right)\left[W^{\prime}\left(x-x^{\prime}\right) f\left(u\left(x^{\prime}, t\right)\right)-W\left(x-x^{\prime}\right) \frac{\partial f\left(u\left(x^{\prime}, t\right)\right)}{\partial x^{\prime}}\right] \mathrm{d} x^{\prime}, \\
\frac{1}{\alpha} \frac{\partial v(x, t)}{\partial t}= & -v(x, t)+u(x, t) .
\end{aligned}
$$

Copyright $@$ by SIAM. Unauthorized reproduction of this article is prohibited. 
Although the inhomogeneous system is not translationally invariant, we can assume that perturbations about the homogeneous system will provide us with nearly translationally invariant solutions [20]. Thus, we perform the change of variables $\xi=x-\phi(t)$ and $\tau=t$ so that (3.3) becomes

$$
\begin{aligned}
\frac{\partial u(\xi, \tau)}{\partial \tau} & =-u(\xi, \tau)+\int_{-\infty}^{\infty} W\left(\xi-\xi^{\prime}\right) f\left(u\left(\xi^{\prime}, \tau\right)\right) \mathrm{d} \xi^{\prime}+\phi^{\prime} \frac{\partial u(\xi, \tau)}{\partial \xi} \\
& +\varepsilon \int_{-\infty}^{\infty} \mathcal{D}\left(\frac{\xi^{\prime}+\phi}{\varepsilon}\right)\left[W^{\prime}\left(\xi-\xi^{\prime}\right) f\left(u\left(\xi^{\prime}, \tau\right)\right)-W\left(\xi-\xi^{\prime}\right) \frac{\partial f\left(u\left(\xi^{\prime}, \tau\right)\right)}{\partial \xi^{\prime}}\right] \mathrm{d} \xi^{\prime}, \\
\text { (3.4) } \frac{1}{\alpha} \frac{\partial v(\xi, \tau)}{\partial \tau} & =-v(\xi, \tau)+u(\xi, \tau)+\frac{\phi^{\prime}}{\alpha} \frac{\partial v(\xi, \tau)}{\partial \xi} .
\end{aligned}
$$

Next perform the perturbation expansions

$$
\begin{aligned}
u(\xi, \tau) & =U(\xi)+\varepsilon u_{1}(\xi, \tau)+\varepsilon^{2} u_{2}(\xi, \tau)+\cdots, \\
v(\xi, \tau) & =V(\xi)+\varepsilon v_{1}(\xi, \tau)+\varepsilon^{2} v_{2}(\xi, \tau)+\cdots, \\
\phi^{\prime}(\tau) & =c+\varepsilon \phi_{1}^{\prime}(\tau)+\varepsilon^{2} \phi_{2}^{\prime}(\tau)+\cdots,
\end{aligned}
$$

where $(U(\xi), V(\xi))^{\mathrm{T}}$ is a traveling pulse solution of the corresponding homogeneous system (see (3.2)) and $c$ is the speed of the unperturbed pulse. The first-order terms $u_{1}, v_{1}$ satisfy

$$
-\frac{\partial}{\partial \tau}\left(\begin{array}{c}
u_{1}(\xi, \tau) \\
v_{1}(\xi, \tau) / \alpha
\end{array}\right)+\mathcal{L}\left(\begin{array}{c}
u_{1}(\xi, \tau) \\
v_{1}(\xi, \tau)
\end{array}\right)=-\phi_{1}^{\prime}(\tau)\left(\begin{array}{c}
U^{\prime}(\xi) \\
V^{\prime}(\xi) / \alpha
\end{array}\right)+\left(\begin{array}{c}
h_{1}\left(\xi, \frac{\phi}{\varepsilon}\right) \\
0
\end{array}\right)
$$

where

$$
\mathcal{L}\left(\begin{array}{l}
u \\
v
\end{array}\right)=\left(\begin{array}{l}
c \frac{d u}{d \xi}-u+\int_{-\infty}^{\infty} W\left(\xi-\xi^{\prime}\right) f^{\prime}\left(U\left(\xi^{\prime}\right)\right) u\left(\xi^{\prime}\right) d \xi^{\prime}-\beta v \\
\frac{c}{\alpha} \frac{d v}{d \xi}-v+u
\end{array}\right)
$$

for $u, v \in \mathcal{C}^{1}(\mathbb{R}, \mathbb{C})$ and

$$
h_{1}\left(\xi, \frac{\phi}{\varepsilon}\right)=-\int_{-\infty}^{\infty} \mathcal{D}\left(\frac{\xi^{\prime}+\phi}{\varepsilon}\right)\left[W^{\prime}\left(\xi-\xi^{\prime}\right) f\left(U\left(\xi^{\prime}\right)\right)-W\left(\xi-\xi^{\prime}\right) \frac{\mathrm{d} f\left(U\left(\xi^{\prime}\right)\right)}{\mathrm{d} \xi^{\prime}}\right] \mathrm{d} \xi^{\prime} .
$$

The linear operator $\mathcal{L}$ has a one-dimensional null-space spanned by $\left(U^{\prime}(\xi), V^{\prime}(\xi)\right)^{\mathrm{T}}$. The existence of $\left(U^{\prime}(\xi), V^{\prime}(\xi)\right)^{\mathrm{T}}$ as a null-vector follows immediately from differentiating the homogeneous equation (3.2) and is a consequence of the translation invariance of the homogeneous system. Uniqueness can be shown using properties of positive linear operators. A bounded solution to (3.9) then exists if and only if the right-hand side is orthogonal to all elements of the null-space of the adjoint operator $\mathcal{L}^{*}$. The latter is defined according to the inner product relation

$$
\int_{-\infty}^{\infty}(a(\xi) b(\xi)) \mathcal{L}\left(\begin{array}{c}
u(\xi) \\
v(\xi)
\end{array}\right) d \xi=\int_{-\infty}^{\infty}(u(\xi) v(\xi)) \mathcal{L}^{*}\left(\begin{array}{c}
a(\xi) \\
b(\xi)
\end{array}\right)
$$

where $u(\xi), v(\xi), a(\xi)$, and $b(\xi)$ are arbitrary integrable functions. It follows that

$$
\mathcal{L}^{*}\left(\begin{array}{c}
a \\
b
\end{array}\right)=\left(\begin{array}{l}
-c \frac{d a}{d \xi}-a+b+f^{\prime}(U(\xi)) \int_{-\infty}^{\infty} W\left(\xi-\xi^{\prime}\right) a\left(\xi^{\prime}\right) d \xi^{\prime} \\
-\frac{c}{\alpha} \frac{d b}{d \xi}-\beta a-b
\end{array}\right)
$$

Copyright (c) by SIAM. Unauthorized reproduction of this article is prohibited. 
The adjoint operator also has a one-dimensional null-space spanned by some vector $(A, B)^{T}$. (An explicit construction of this null-vector in the case of a Heaviside nonlinearity will be presented in section 4.) Therefore, for (3.9) to have a solution, it is necessary that

$$
K \phi_{1}^{\prime}(\tau)=\int_{-\infty}^{\infty} A(\xi) h_{1}\left(\xi, \frac{\phi}{\varepsilon}\right) d \xi
$$

where

$$
K=\int_{-\infty}^{\infty}\left[A(\xi) U^{\prime}(\xi)+\alpha^{-1} B(\xi) V^{\prime}(\xi)\right] d \xi
$$

Substituting for $h_{1}$ using (3.10) leads to a first-order differential equation for the phase $\phi$ :

$$
\frac{d \phi}{d \tau}=c-\varepsilon \Phi_{1}\left(\frac{\phi}{\varepsilon}\right)
$$

where

$$
\begin{aligned}
\Phi_{1}\left(\frac{\phi}{\varepsilon}\right)= & \frac{1}{K} \int_{-\infty}^{\infty} \int_{-\infty}^{\infty} A(\xi) \mathcal{D}\left(\frac{\xi^{\prime}+\phi}{\varepsilon}\right) \\
& \times\left[W^{\prime}\left(\xi-\xi^{\prime}\right) f\left(U\left(\xi^{\prime}\right)\right)-W\left(\xi-\xi^{\prime}\right) \frac{d f\left(U\left(\xi^{\prime}\right)\right)}{d \xi^{\prime}}\right] d \xi^{\prime} d \xi
\end{aligned}
$$

If the right-hand side of (3.15) is strictly positive, then there exists a traveling pulse of the approximate form $U(x-\phi(t))$ and of average speed $\bar{c}=2 \pi \varepsilon / T$ with

$$
T=\int_{0}^{2 \pi \varepsilon} \frac{d \phi}{c-\varepsilon \Phi_{1}(\phi / \varepsilon)} .
$$

However, if the right-hand side of (3.15) vanishes for some $\phi$, then the first-order analysis predicts wave propagation failure.

4. Calculation of average wave speed. In this section we use (3.17) to calculate the average wave speed $\bar{c}$ as a function of $\varepsilon$ in the limiting case of a Heaviside nonlinearity. Note that since derivatives of $f$ always appear inside integral terms, the high gain limit $\eta \rightarrow \infty$ is well defined. One advantage of using a Heaviside nonlinearity is that all calculations can be carried out explicitly. Moreover, as previously shown for traveling fronts [3], in the case of smooth nonlinearities it is necessary to develop the perturbation analysis to $\mathcal{O}\left(\varepsilon^{2}\right)$ since the $\mathcal{O}(\varepsilon)$ terms may be exponentially small; see also section 4.3 .

4.1. Homogeneous network with Heaviside nonlinearity. The existence (and stability) of single bump traveling pulse solutions in the homogeneous network obtained by setting $f=H$ and $w\left(x, x^{\prime}\right)=W\left(\left|x-x^{\prime}\right|\right)$ in $(2.1)$ has been studied by a number of authors [31, 33, 42, 10, 14]. A single bump solution $(U(\xi), V(\xi))$ is one for which $U$ is above threshold over a domain of length $a$, corresponding to the width of the bump, and subthreshold everywhere else. In other words, the activity $U$ crosses threshold at only two points, which by translation invariance can be taken to be $\xi=-a, 0$ :

$$
\begin{array}{rlrl}
U(-a)= & U(0)=\kappa ; & U(\xi) \longrightarrow 0 \text { as } \xi \longrightarrow \pm \infty ; \\
U(\xi)>\kappa,-a<\xi<0 ; & U(\xi)<\kappa \quad \text { otherwise. }
\end{array}
$$


It follows from (3.2) with $f=H$ that

$$
\begin{aligned}
-c U_{\xi} & =-U-\beta V+\int_{-a}^{0} W(\xi-\eta) d \eta, \\
-\frac{c}{\alpha} V_{\xi} & =-V+U .
\end{aligned}
$$

One way to solve this pair of equations is to use variation of parameters $[42,14]$. For completeness, we present the details of this calculation here, since some of the results will be used in our subsequent analysis.

Let $\mathbf{s}=(U, V)^{T}$ and rewrite the system as

$$
\mathcal{L} \mathbf{s} \equiv\left(\begin{array}{c}
c U^{\prime}(\xi)-U(\xi)-\beta V(\xi) \\
c V^{\prime}(\xi)+\alpha U(\xi)-\alpha V(\xi)
\end{array}\right)=-\left(\begin{array}{c}
N_{e}(\xi) \\
0
\end{array}\right)
$$

where

$$
N_{e}(\xi)=\Omega(\xi+a)-\Omega(\xi), \quad \Omega(\xi)=\int_{-\infty}^{\xi} W\left(\xi^{\prime}\right) d \xi^{\prime}
$$

We solve (4.2) using variation of parameters. The homogeneous problem $\mathcal{L} \mathbf{s}=\mathbf{0}$ has two linearly independent solutions,

$$
\mathbf{S}_{+}(\xi)=\left(\begin{array}{c}
\beta \\
m_{+}-1
\end{array}\right) \exp \left(\mu_{+} \xi\right), \quad \mathbf{S}_{-}(\xi)=\left(\begin{array}{c}
\beta \\
m_{-}-1
\end{array}\right) \exp \left(\mu_{-} \xi\right)
$$

where

$$
\mu_{ \pm}=\frac{m_{ \pm}}{c}, \quad m_{ \pm}=\frac{1}{2}\left(1+\alpha \pm \sqrt{(1-\alpha)^{2}-4 \mu \beta}\right) .
$$

We shall work in the parameter regime where $\mu_{ \pm}$are real, though interesting behavior can arise when $\mu_{ \pm}$is complex [38]. Thus, set

$$
\mathbf{s}(\xi)=\left[\mathbf{S}_{+} \mid \mathbf{S}_{-}\right]\left(\begin{array}{c}
a(\xi) \\
b(\xi)
\end{array}\right)
$$

where $a, b \in \mathcal{C}^{1}(\mathbb{R}, \mathbb{R})$, and $[A \mid B]$ denotes the matrix whose first column is $A$ and whose second column is $B$. Since $\mathcal{L} \mathbf{S}_{ \pm}=0$, (4.2) becomes

$$
\left[\mathbf{S}_{+} \mid \mathbf{S}_{-}\right] \frac{\partial}{\partial \xi}\left(\begin{array}{c}
a(\xi) \\
b(\xi)
\end{array}\right)=-\frac{1}{c}\left(\begin{array}{c}
N_{e}(\xi) \\
0
\end{array}\right) .
$$

Since $\left[\mathbf{S}_{+} \mid \mathbf{S}_{-}\right]$is invertible, we find that

$$
\frac{\partial}{\partial \xi}\left(\begin{array}{c}
a(\xi) \\
b(\xi)
\end{array}\right)=-\frac{1}{c \beta\left(m_{+}-m_{-}\right)}\left[\mathbf{Z}_{+} \mid \mathbf{Z}_{-}\right]^{T}\left(\begin{array}{c}
N_{e}(\xi) \\
0
\end{array}\right)
$$

where

$$
\mathbf{Z}_{+}(\xi)=\left(\begin{array}{c}
1-m_{-} \\
\beta
\end{array}\right) \exp \left(-\mu_{+} \xi\right), \quad \mathbf{Z}_{-}(\xi)=-\left(\begin{array}{c}
1-m_{+} \\
\beta
\end{array}\right) \exp \left(-\mu_{-} \xi\right)
$$

Copyright $@$ by SIAM. Unauthorized reproduction of this article is prohibited. 
For $c>0$, we can integrate from $\xi$ to $\infty$ and find

$$
\left(\begin{array}{c}
a(\xi) \\
b(\xi)
\end{array}\right)=\left(\begin{array}{c}
a_{\infty} \\
b_{\infty}
\end{array}\right)+\frac{1}{c \beta\left(m_{+}-m_{-}\right)} \int_{\xi}^{\infty}\left[\mathbf{Z}_{+} \mid \mathbf{Z}_{-}\right]^{T}\left(\begin{array}{c}
N_{e}\left(\xi^{\prime}\right) \\
0
\end{array}\right) d \xi^{\prime}
$$

where $a_{\infty}, b_{\infty}$ are the values of $a(\xi), b(\xi)$ as $\xi \rightarrow \infty$. Thus

$$
\mathbf{s}(\xi)=\left[\mathbf{S}_{+} \mid \mathbf{S}_{-}\right]\left(\begin{array}{c}
a_{\infty} \\
b_{\infty}
\end{array}\right)+\frac{1}{c \beta\left(m_{+}-m_{-}\right)}\left[\mathbf{S}_{+} \mid \mathbf{S}_{-}\right] \int_{\xi}^{\infty}\left[\mathbf{Z}_{+} \mid \mathbf{Z}_{-}\right]^{T}\left(\begin{array}{c}
N_{e}\left(\xi^{\prime}\right) \\
0
\end{array}\right) d \xi^{\prime}
$$

Using Hölder's inequality and that $N_{e} \in \mathcal{C}^{0}(\mathbb{R}, \mathbb{R})$, we can show that the integral in (4.6) is bounded for all $\xi \in R$. Thus, a bounded solution $\mathbf{s}$ exists if $a_{\infty}=b_{\infty}=0$. Our general traveling pulse solution is given by

$$
\mathbf{s}(\xi)=\frac{1}{c \beta\left(m_{+}-m_{-}\right)}\left[\mathbf{S}_{+} \mid \mathbf{S}_{-}\right] \int_{\xi}^{\infty}\left[\mathbf{Z}_{+} \mid \mathbf{Z}_{-}\right]^{T}\left(\begin{array}{c}
N_{e}\left(\xi^{\prime}\right) \\
0
\end{array}\right) d \xi^{\prime} .
$$

Furthermore, if we define

$$
\mathcal{M}_{ \pm}(\xi)=\frac{1}{c\left(m_{+}-m_{-}\right)} \int_{\xi}^{\infty} \mathrm{e}^{\mu_{ \pm}\left(\xi-\xi^{\prime}\right)} N_{e}\left(\xi^{\prime}\right) d \xi^{\prime}
$$

we can express our solution $(U, V)$ as

$$
\begin{aligned}
& U(\xi)=\left(1-m_{-}\right) \mathcal{M}_{+}(\xi)-\left(1-m_{+}\right) \mathcal{M}_{-}(\xi) \\
& V(\xi)=\beta^{-1}\left(m_{+}-1\right)\left(1-m_{-}\right)\left[\mathcal{M}_{+}(\xi)-\mathcal{M}_{-}(\xi)\right] .
\end{aligned}
$$

Since $N_{e}(\xi)$ is dependent upon the pulse width $a$, the threshold conditions $U(-a)=U(0)=\kappa$ lead to the following consistency conditions for the existence of a traveling pulse:

$$
\begin{aligned}
& \kappa=\left(1-m_{-}\right) \mathcal{M}_{+}(-a)-\left(1-m_{+}\right) \mathcal{M}_{-}(-a), \\
& \kappa=\left(1-m_{-}\right) \mathcal{M}_{+}(0)-\left(1-m_{+}\right) \mathcal{M}_{-}(0) .
\end{aligned}
$$

This pair of nonlinear equations determines the pulse width $a$ and wave speed $c$ of a single bump traveling wave solution as a function of the various parameters of the network. For a given weight distribution $W(x)$, existence of such a solution is established if a solution for $a, c$ can be found, and provided that $U$ does not cross threshold at any other points besides $\xi=-a, 0$. Recently the existence (and stability) of single bump traveling waves has been examined for quite a general class of weight distributions [32] which includes both exponential and Gaussian distributions. For concreteness, we consider the exponential weight function (2.5) with $W_{0}=d=1$. Numerically solving (4.9) and (4.10) for $a$ and $c$ as a function of the adaptation rate $\alpha$ yields the existence curves shown in Figure 2. This figure illustrates the well-known result that for sufficiently slow adaptation $(\operatorname{small} \alpha)$ there exists a pair of traveling pulses with the fast/wide pulse stable and the slow/narrow pulse unstable [31]. Also shown in the figure is the stability of the various solution branches, which can be determined analytically using an Evans function approach [42, 10, 14, 32].

Copyright (c) by SIAM. Unauthorized reproduction of this article is prohibited. 

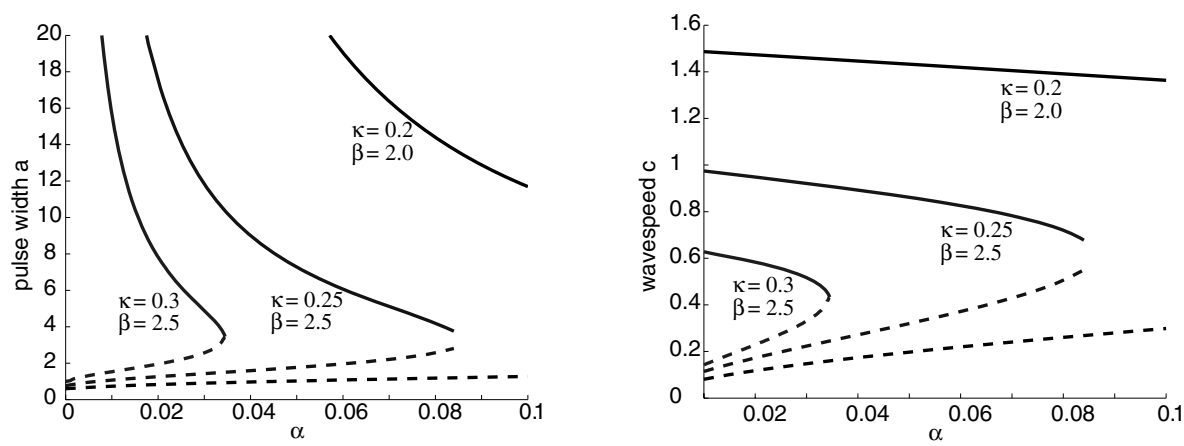

Figure 2. Existence curves for a single bump traveling pulse solution of (2.1) in the case of a homogeneous network with an exponential weight distribution $W(x)=\mathrm{e}^{-|x| / 2}$. Left: Existence curves in the $(\alpha, a)$ plane. Right: Existence curves in the $(\alpha, c)$ plane. Pulses exist only for small enough $\alpha$ (sufficiently slow adaptation). For each parameter set, there exists a stable branch (solid) of wide/fast pulses and an unstable branch (dashed) of narrow/slow pulses. In the case $\kappa=0.3$ the branches annihilate at a saddle-node bifurcation at a critical value $\alpha_{c}$. In the other two cases, the branches end abruptly due to the fact that the eigenvalues $\mu_{ \pm}$become complex-valued [38].
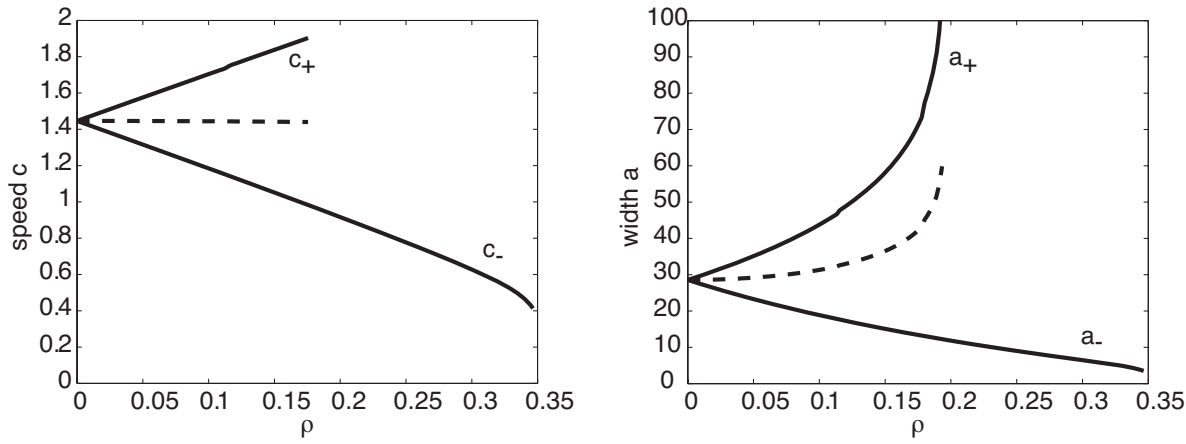

Figure 3. Existence curves for a single bump traveling pulse solution of (2.1) in the case of a homogeneous network with an exponential weight distribution $W(x)=W_{ \pm} \mathrm{e}^{-|x| / 2}$ with $W_{ \pm}=1 \pm \rho$. Left: Plot of wave speed $c_{ \pm}$as a function of $\rho$ for weight amplitudes $W_{ \pm}$. The dashed curve indicates the arithmetic mean of pair $c_{ \pm}$. The slower branch terminates at around $\rho=0.35$ due to a saddle-node bifurcation. The faster branch terminates due to a blow-up of the pulse width. Right: Plot of pulse width $a_{ \pm}$as a function of $\rho$ for weight amplitudes $W_{ \pm}$.

The analysis of existence in a homogeneous network also provides some insight into what happens when we include a periodic modulation of the weights according to (2.4) and (2.6). Such a modulation induces a periodic variation in the amplitude $W_{0}$ of the exponential weight distribution (2.5) between the limiting values $W_{ \pm}=(1 \pm \rho) W_{0}$. This suggests that the speed of a wave in the inhomogeneous network will be bounded by the speeds $c_{ \pm}$of a traveling wave in the corresponding homogeneous network obtained by taking $W_{0} \rightarrow W_{ \pm}$. Note that rescaling the weight distribution in (4.9) and (4.10) is equivalent to rescaling the threshold according to $\kappa \rightarrow \kappa /(1 \pm \rho)$. In Figure 3 we plot the speeds $c_{ \pm}$and the corresponding pulse widths $a_{ \pm}$as a function of $\rho$. For sufficiently small $\rho$, the wave speed $c_{+}$increases with $\rho$ at approximately the same rate as $c_{-}$decreases so that their arithmetic mean remains constant. Therefore, one might expect that a periodic variation in weights would lead to a corresponding periodic 
variation in wave speed such that the mean wave speed is approximately independent of $\rho$. However, when a pulse enters a region of enhanced synaptic weights, the resulting increase in wave speed coincides with a rapid increase in pulse width as a function of $\rho$. Thus, the pulse will tend to extend into neighboring regions of reduced synaptic weights and the resulting spatial averaging will counteract the speeding up of the wave. On the other hand, when a pulse enters a region of reduced synaptic weights, the reduction in wave speed coincides with a reduction in pulse width so that spatial averaging can no longer be carried out as effectively. (The effectiveness of spatial averaging will depend on the ratio of the pulse width $a$ to the periodicity $2 \pi \varepsilon$ of the weight modulation.) Hence, we expect regions where the weights are reduced to have more effect on wave propagation than regions where the weights are enhanced, suggesting that a periodic weight modulation leads to slower, narrower waves. This is indeed found to be the case, both in our perturbation analysis (section 4.2) and in our numerical simulations (section 5). Interestingly, we also find that traveling waves persist for larger values of $\rho$ than predicted by our analysis of single bumps in homogeneous networks, although such waves tend to consist of multiple bumps (see section 5).

4.2. Inhomogeneous network with Heaviside nonlinearity. Suppose that the homogeneous network with a Heaviside nonlinearity supports a stable traveling wave solution $(U(\xi), V(\xi))^{T}$ of wave speed $c$. As shown in section 4.1, a stable/unstable pair of traveling waves exists for sufficiently slow adaptation. In order to calculate the average wave speed $\bar{c}$ for nonzero $\varepsilon$ and $\rho$ (see (3.17)), we first need to compute the null-vector $(A(\xi), B(\xi))^{T}$ of the adjoint operator $\mathcal{L}^{*}$ defined by (3.12). In the case of a Heaviside nonlinearity,

$$
\begin{aligned}
-c \frac{d A(\xi)}{d \xi}-A(\xi)+B(\xi) & +\frac{\delta(\xi)}{\left|U^{\prime}(0)\right|} \int_{-\infty}^{\infty} W\left(\xi-\xi^{\prime}\right) A\left(\xi^{\prime}\right) d \xi^{\prime} \\
+\frac{\delta(\xi+a)}{\left|U^{\prime}(-a)\right|} \int_{-\infty}^{\infty} W\left(\xi-\xi^{\prime}\right) A\left(\xi^{\prime}\right) d \xi^{\prime} & =0 \\
& -\frac{c}{\alpha} \frac{d B(\xi)}{d \xi}-\beta A(\xi)-B(\xi)=0 .
\end{aligned}
$$

For $\xi \neq 0,-a$, this has solutions of the form $\left(A(\xi), B(\xi)^{T}\right)=\mathbf{u} e^{-\lambda \xi}$ with associated characteristic equation $\mathbf{M u}=c \lambda \mathbf{u}$ and

$$
\mathbf{M}=\left(\begin{array}{cc}
1 & -1 \\
\beta \alpha & \alpha
\end{array}\right)
$$

The eigenvalues are $\lambda=\mu_{ \pm}=m_{ \pm} / c$ with $m_{ \pm}$given by (4.4). The corresponding eigenvectors are

$$
\mathbf{u}_{ \pm}=\left(\begin{array}{c}
1 \\
1-m_{ \pm}
\end{array}\right)
$$

The presence of the Dirac delta functions at $\xi=0,-a$ then suggests that we take the nullsolution to be of the form

$$
\begin{aligned}
\mathbf{V}^{*}(\xi)= & \gamma_{+} \mathbf{u}_{+}\left[e^{-\mu_{+} \xi} \Theta(\xi)+\chi e^{-\mu_{+}(\xi+a)} \Theta(\xi+a)\right] \\
& +\gamma_{-} \mathbf{u}_{-}\left[e^{-\mu_{-} \xi} \Theta(\xi)+\chi e^{-\mu_{-}(\xi+a)} \Theta(\xi+a)\right]
\end{aligned}
$$

Copyright $@$ by SIAM. Unauthorized reproduction of this article is prohibited. 
with the coefficients $\gamma_{ \pm}$chosen such that the Dirac delta function terms that come from differentiating the null-vector appear only in the $A(\xi)$ term,

$$
\gamma_{+} \mathbf{u}_{+}+\gamma_{-} \mathbf{u}_{-}=\left(\begin{array}{c}
\Gamma \\
0
\end{array}\right)
$$

and $\chi$ is a constant yet to be determined. Taking

$$
\gamma_{ \pm}= \pm\left(1-m_{\mp}\right)
$$

we have $\Gamma=m_{+}-m_{-}$.

In order to determine $\chi$, substitute (4.14) into (4.11) to obtain the pair of equations

$$
c\left(m_{+}-m_{-}\right)=\frac{1}{\left|U^{\prime}(0)\right|}(\Lambda(0)+\chi \Lambda(-a))
$$

and

$$
\chi c\left(m_{+}-m_{-}\right)=\frac{1}{\left|U^{\prime}(-a)\right|}(\Lambda(a)+\chi \Lambda(0))
$$

with

$$
\Lambda(\zeta)=\int_{0}^{\infty}\left[\left(1-m_{-}\right) W(\xi+\zeta) e^{-\mu_{+} \xi}-\left(1-m_{+}\right) W(\xi+\zeta) e^{-\mu_{-} \xi}\right] d \xi .
$$

We require that (4.17) and (4.18) be consistent with the formula for $U^{\prime}(\xi)$ obtained by differentiating (4.7) with respect to $\xi$ :

$$
\begin{aligned}
U^{\prime}(\xi)= & \frac{1-m_{-}}{c\left(m_{+}-m_{-}\right)} \int_{\xi}^{\infty} e^{\mu_{+}\left(\xi-\xi^{\prime}\right)}\left[W\left(\xi^{\prime}+a\right)-W\left(\xi^{\prime}\right)\right] d \xi^{\prime} \\
& -\frac{1-m_{+}}{c\left(m_{+}-m_{-}\right)} \int_{\xi}^{\infty} e^{\mu_{-}\left(\xi-\xi^{\prime}\right)}\left[W\left(\xi^{\prime}+a\right)-W\left(\xi^{\prime}\right)\right] d \xi^{\prime} .
\end{aligned}
$$

It follows that

$$
\left|U^{\prime}(0)\right|=-U^{\prime}(0)=\frac{\Lambda(0)-\Lambda(a)}{c\left(m_{+}-m_{-}\right)}, \quad\left|U^{\prime}(-a)\right|=U^{\prime}(-a)=\frac{\Lambda(0)-\Lambda(-a)}{c\left(m_{+}-m_{-}\right)},
$$

which, together with (4.17) and (4.18), imply

$$
\Lambda(0)-\Lambda(a)=\Lambda(0)+\chi \Lambda(-a), \quad \chi(\Lambda(0)-\Lambda(-a))=\Lambda(a)+\chi \Lambda(0) .
$$

Hence, (4.14) is a solution provided that

$$
\chi=-\frac{\Lambda(a)}{\Lambda(-a)} .
$$

This is also a constructive proof that the adjoint linear operator $\mathcal{L}^{*}$ for a Heaviside nonlinearity has a one-dimensional null-space spanned by $\mathbf{V}^{*}$.

Copyright (c) by SIAM. Unauthorized reproduction of this article is prohibited. 
Having found the null-solution (4.14), we now determine the phase function $\Phi_{1}$ given by (3.16) with $f=H$. First, the constant $K$ of (3.14) is evaluated by substituting for $(A(\xi), B(\xi))$ using (4.14) and substituting for $(U(\xi), V(\xi))$ using (4.7) and (4.8). The rather lengthy expression for $K$ is given in the appendix. Next, we evaluate the double integral on the right-hand side of (3.16) by setting $\mathcal{D}(x)=\mathrm{e}^{i x}$ and using Fourier transforms. This gives

$$
K \Phi_{1}\left(\frac{\phi}{\varepsilon}\right)=\frac{i}{\varepsilon} \mathrm{e}^{i \phi / \varepsilon} \int_{-\infty}^{\infty} W(x)\left[\int_{-\infty}^{\infty} \mathrm{e}^{i q x} \widetilde{A}^{*}(q) \widetilde{f(U)}\left(q+\varepsilon^{-1}\right) \frac{\mathrm{d} q}{2 \pi}\right] d x
$$

where $^{*}$ denotes complex conjugate and

$$
\widetilde{A}(q)=\int_{-\infty}^{\infty} \mathrm{e}^{i q x} A(x) \mathrm{d} x .
$$

In the case of a Heaviside nonlinearity and a pulse of width $a, f(U(\xi))=\Theta(\xi+a)-\Theta(\xi)$, and $A(x)$ is given explicitly by the first component of the null-vector in (4.14). Taking Fourier transforms of these expressions shows that

$$
\widetilde{A}(q)=-\left(1+\chi \mathrm{e}^{-i q a}\right)\left[\frac{\gamma_{+}}{i q-\mu_{+}}+\frac{\gamma_{-}}{i q-\mu_{-}}\right], \quad \widetilde{f(U)}(q)=\frac{1-\mathrm{e}^{-i q a}}{i q-0^{+}} .
$$

If these Fourier transforms are now substituted into (4.22), we have

$$
\begin{aligned}
K \Phi_{1}\left(\frac{\phi}{\varepsilon}\right)= & \frac{\mathrm{e}^{i \phi / \varepsilon}}{\varepsilon} \int_{-\infty}^{\infty} W(x)\left[\int _ { - \infty } ^ { \infty } \left\{\frac{\gamma_{+}\left(1-\mathrm{e}^{-i\left(q+\varepsilon^{-1}\right) a}+\chi \mathrm{e}^{i q a}-\chi \mathrm{e}^{-i a / \varepsilon}\right) \mathrm{e}^{i q x}}{\left(q+\varepsilon^{-1}+i 0^{+}\right)\left(q-i \mu_{+}\right)}\right.\right. \\
& \left.\left.+\frac{\gamma_{-}\left(1-\mathrm{e}^{-i\left(q+\varepsilon^{-1}\right) a}+\chi \mathrm{e}^{i q a}-\chi \mathrm{e}^{-i a / \varepsilon}\right) \mathrm{e}^{i q x}}{\left(q+\varepsilon^{-1}+i 0^{+}\right)\left(q-i \mu_{-}\right)}\right\} \frac{\mathrm{d} q}{2 \pi i}\right] \mathrm{d} x .
\end{aligned}
$$

The resulting integral over $q$ can be evaluated by closing the contour in the upper-half or lower-half complex $q$-plane depending on the sign of $x, x \pm a$. We find that there are only contributions from the poles at $q=i \mu_{ \pm}$with $\mu_{ \pm}>0$, whereas there is a removable singularity at $q=-\varepsilon^{-1}-i 0^{+}$. Thus

$$
\begin{aligned}
K \Phi_{1}\left(\frac{\phi}{\varepsilon}\right)= & \frac{\gamma_{+} \mathrm{e}^{i \phi / \varepsilon}}{\varepsilon\left(\varepsilon^{-1}+i \mu_{+}\right)}\left[\left(1-\chi \mathrm{e}^{-i a / \varepsilon}\right) \widehat{\Omega}_{+}(0)+\chi \widehat{\Omega}_{+}(-a)-\mathrm{e}^{-i a / \varepsilon} \widehat{\Omega}_{+}(a)\right] \\
& +\frac{\gamma_{-} \mathrm{e}^{i \phi / \varepsilon}}{\varepsilon\left(\varepsilon^{-1}+i \mu_{-}\right)}\left[\left(1-\chi \mathrm{e}^{-i a / \varepsilon}\right) \widehat{\Omega}_{-}(0)+\chi \widehat{\Omega}_{-}(-a)-\mathrm{e}^{-i a / \varepsilon} \widehat{\Omega}_{-}(a)\right],
\end{aligned}
$$

with

$$
\widehat{\Omega}_{ \pm}(s)=\int_{0}^{\infty} W(x+s) \mathrm{e}^{-\mu_{ \pm} x} \mathrm{~d} x .
$$

Taking the imaginary part of the above equation then determines the phase function $K \Phi_{1}$ for $\mathcal{D}(x)=\rho \sin (x)$. After a straightforward calculation, we find that

$$
\begin{aligned}
\frac{K}{\rho} \Phi_{1}\left(\frac{\phi}{\varepsilon}\right)= & \left(\Xi_{+}+\Xi_{-}\right) \sin \left(\frac{\phi}{\varepsilon}\right)+\left(\Pi_{+}+\Pi_{-}\right) \sin \left(\frac{\phi-a}{\varepsilon}\right) \\
& +\left(\Upsilon_{+}+\Upsilon_{-}\right) \cos \left(\frac{\phi}{\varepsilon}\right)+\left(\Psi_{+}+\Psi_{-}\right) \cos \left(\frac{\phi-a}{\varepsilon}\right),
\end{aligned}
$$

where the explicit expressions for $\Xi_{ \pm}, \Pi_{ \pm}, \Upsilon_{ \pm}, \Psi_{ \pm}$are given in the appendix. 

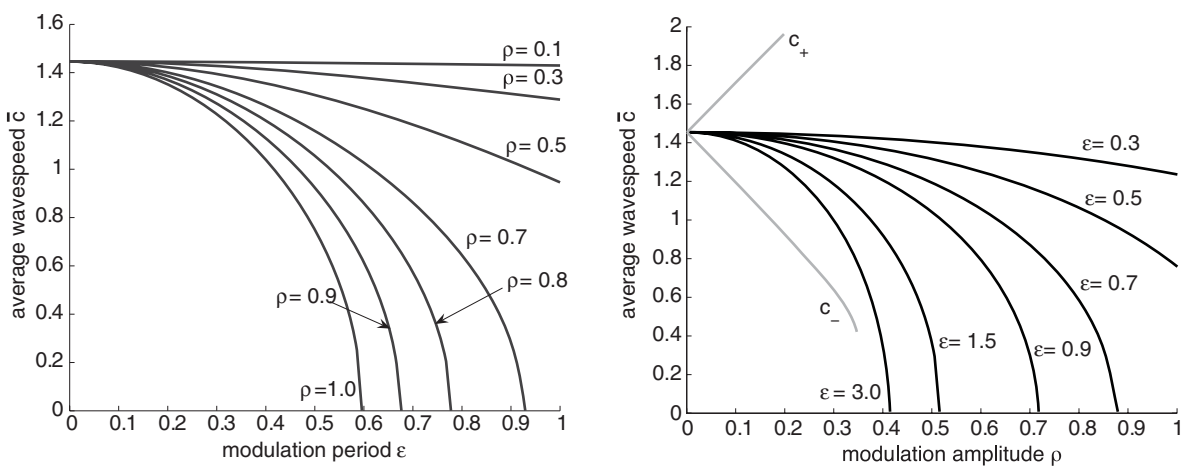

Figure 4. Left: Average wave speed $\bar{c}$ versus $\varepsilon$ for various values of the modulation amplitude $\rho$. The critical value of $\varepsilon$ for wave propagation failure decreases as $\rho$ increases. Right: Average wave speed $\bar{c}$ versus $\rho$ for various values of the modulation period $\varepsilon$. For the sake of comparison, the speed curves previously plotted in Figure 3 for a homogeneous network are also shown (gray curves). Other parameters are $\kappa=0.2, \alpha=0.04$, and $\beta=2.0$.

Finally, we numerically calculate the average wave speed $\bar{c}$ by substituting (4.28) into (3.17). Note that we use the exact expression for $\Phi_{1}$ that includes all higher-order terms in $\varepsilon$, rather than keeping only the $\mathcal{O}(1)$ term, since this gives a better estimate of the wave speed. In Figure 4 we show some example plots of $\bar{c}$ as a function of $\varepsilon$ and $\rho$. It can be seen that for each choice of parameters, $\bar{c}$ is a monotonically decreasing function of $\varepsilon$ and $\rho$, with $\bar{c}$ approaching the speed $c$ of the homogeneous wave in the limits $\varepsilon \rightarrow 0$ and $\rho \rightarrow 0$. Hence, although the periodic modulation enhances the strength of connections in some regions and reduces them in others compared to the homogeneous case (see Figure 1), the net result is an effective reduction in wave speed. This is consistent with our discussion of Figure 3 in section 4.1, where we used a spatial averaging argument combined with the observation that faster waves are wider to infer that regions of reduced synaptic weights affect wave propagation more than regions of enhanced weights. Figure 4 also suggests that for sufficiently small $\varepsilon$ there exists a traveling wave solution for all $\rho, 0 \leq \rho<1$, whereas for larger values of $\varepsilon$ there is a critical value $\rho_{c}$ beyond which propagation failure occurs. That is, $\bar{c} \rightarrow 0$ as $\rho \rightarrow \rho_{c}$, and this critical value decreases as the periodicity $\varepsilon$ of the inhomogeneity increases. Similarly, for sufficiently large $\rho$ there exists a critical period $\varepsilon_{c}$ such that $\bar{c} \rightarrow 0$ as $\varepsilon \rightarrow \varepsilon_{c}$. Analogous results were previously obtained for traveling fronts in a scalar equation [3]. It is important to bear in mind that the calculation of $\bar{c}$ is based on the $\mathcal{O}(\varepsilon)$ perturbation analysis of section 3 , although we do include higher-order terms in the calculation of $\Phi_{1}$. This raises the important question as to whether or not our analysis correctly predicts wave propagation failure in the full system, given that $\bar{c}$ tends to approach zero at relatively large values of $\varepsilon$ and $\rho$. Moreover, the perturbation analysis does not determine the stability of the wave so that propagation failure could occur due to destabilization of the wave for $\rho<\rho_{c}$ or $\varepsilon<\varepsilon_{c}$. This will indeed turn out to be the case as we show in section 5 , where we present numerical solutions of (2.1) and provide further insight into the mechanism for propagation failure.

4.3. Smooth nonlinearities and higher-order corrections. In the case of smooth nonlinearities, the Fourier transforms $\widetilde{A}(q)$ and $\widetilde{f(U)}(q)$ appearing in (4.22) no longer have simple 
poles, and in general $\Phi_{1}$ will consist of exponentially small terms. It follows that $\Phi_{1}$ may be less significant than the $\mathcal{O}\left(\varepsilon^{2}\right)$ terms ignored in the perturbation expansion of (3.4). Therefore, following the treatment of traveling fronts [3], we carry out a perturbation expansion of system (3.4) to $\mathcal{O}\left(\varepsilon^{2}\right)$. This yields an equation for $\left(u_{2}, v_{2}\right)$ of the form

$$
\begin{aligned}
-\frac{\partial}{\partial \tau}\left(\begin{array}{c}
u_{2}(\xi, \tau) \\
v_{2}(\xi, \tau) / \alpha
\end{array}\right)+\mathcal{L}\left(\begin{array}{c}
u_{2}(\xi, \tau) \\
v_{2}(\xi, \tau)
\end{array}\right)= & -\phi_{2}^{\prime}(\tau)\left(\begin{array}{c}
U^{\prime}(\xi) \\
V^{\prime}(\xi) / \alpha
\end{array}\right) \\
& -\phi_{1}^{\prime}(\tau)\left(\begin{array}{c}
u_{1}^{\prime}(\xi) \\
v_{1}^{\prime}(\xi) / \alpha
\end{array}\right)+\left(\begin{array}{c}
h_{2}\left(\xi, \frac{\phi}{\varepsilon}\right) \\
0
\end{array}\right)
\end{aligned}
$$

where $\mathcal{L}$ is defined by (3.9) and

$$
\begin{aligned}
h_{2}\left(\xi, \frac{\phi}{\varepsilon}\right)= & -\frac{1}{2} \int_{-\infty}^{\infty} W\left(\xi-\xi^{\prime}\right) f^{\prime \prime}\left(U\left(\xi^{\prime}\right)\right)\left[u_{1}\left(\xi^{\prime}\right)\right]^{2} \mathrm{~d} \xi^{\prime} \\
& -\int_{-\infty}^{\infty} \mathcal{D}\left(\frac{\left[\xi^{\prime}+\phi\right]}{\varepsilon}\right) W^{\prime}\left(\xi-\xi^{\prime}\right) f^{\prime}\left(U\left(\xi^{\prime}\right)\right) u_{1}\left(\xi^{\prime}\right) \mathrm{d} \xi^{\prime} \\
& +\int_{-\infty}^{\infty} \mathcal{D}\left(\frac{\left[\xi^{\prime}+\phi\right]}{\varepsilon}\right) W\left(\xi-\xi^{\prime}\right)\left[f^{\prime}\left(U\left(\xi^{\prime}\right)\right) u_{1}^{\prime}\left(\xi^{\prime}\right)+f^{\prime \prime}\left(U\left(\xi^{\prime}\right)\right) U^{\prime}\left(\xi^{\prime}\right) u_{1}(\xi)\right] \mathrm{d} \xi^{\prime} .
\end{aligned}
$$

The existence of a bounded solution requires the solvability conditions (3.13) and

$$
K \phi_{2}^{\prime}(\tau)+L(\tau) \phi_{1}^{\prime}(\tau)=\int_{-\infty}^{\infty} A(\xi) h_{2}\left(\xi, \frac{\phi}{\varepsilon}\right) \mathrm{d} \xi
$$

where

$$
L(\tau)=\int_{-\infty}^{\infty}\left[A(\xi) \frac{\partial u_{1}(\xi, \tau)}{\partial \xi}+\alpha^{-1} B(\xi) \frac{\partial v_{1}(\xi, \tau)}{\partial \xi}\right] \mathrm{d} \xi
$$

In order to evaluate the solvability condition (4.31), we must first determine $u_{1}(\xi, \phi / \varepsilon)$ from (3.8). If we choose $\mathcal{D}(x)$ to be a sinusoid, then $u_{1}(\xi, \phi / \varepsilon)$ will include terms that are proportional to $\sin (\phi / \varepsilon)$ and $\cos (\phi / \varepsilon)$. Thus substituting $u_{1}(\xi / \phi / \varepsilon)$ into (4.30) will generate terms of the form $\sin ^{2}(\phi / \varepsilon)$ and $\cos ^{2}(\phi / \varepsilon)$ due to the quadratic term in $u_{1}$. Using the identities $2 \sin ^{2}(x)=1-\cos (2 x)$ and $2 \cos ^{2}(x)=1+\cos (2 x)$ implies that there will be an $\varepsilon$-independent contribution to $\phi_{2}^{\prime}$. Thus for smooth nonlinearities we find that

$$
\frac{\mathrm{d} \phi}{\mathrm{d} \tau}=c+\varepsilon^{2} C_{2}(c)+D_{2}\left(c, \frac{\phi}{\varepsilon}\right)
$$

where $C_{2}$ is independent of $\varepsilon$ and $D_{2}$ is exponentially small in $\varepsilon$. Equation (4.33) is the secondorder version of the phase equation (3.15) in cases where the first-order term is exponentially small. Again, the condition for wave propagation failure is that the right-hand side of (4.33) vanishes for some $\phi$. 

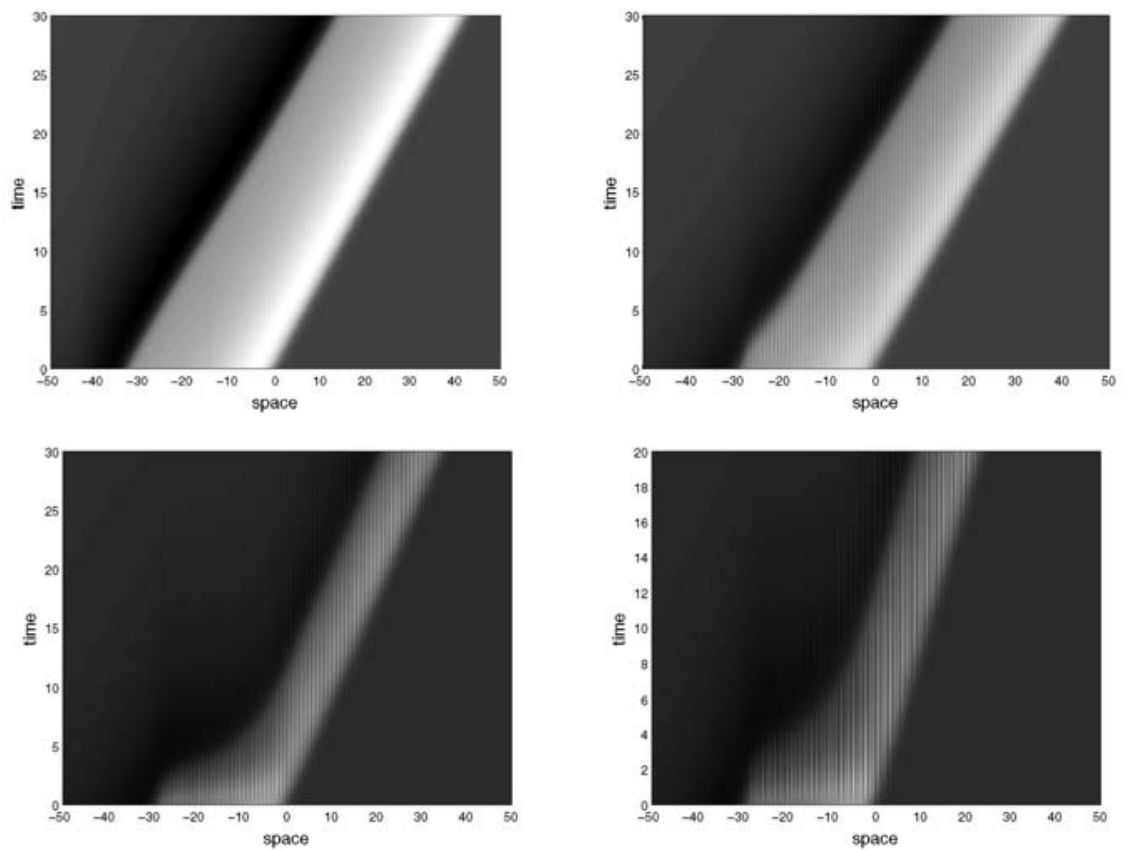

Figure 5. Top left: Stable traveling pulse for a homogeneous network with exponential weight function (2.5) and fixed parameters $\kappa=0.2, \beta=2.0$, and $\alpha=0.04$ (for all plots). Top right: Corresponding traveling pulse for an inhomogeneous network with weight distribution (2.4) and a sinusoidal modulation with $\varepsilon=0.1$ and $\rho=0.3$. We see rippling in the interior of the pulse. Bottom left: Using a more severe inhomogeneity, $\rho=0.8$, leads to rippling in the active region of the pulse such that now the interior crosses below threshold periodically. Bottom right: For $\rho=1$, the effect is even more severe.

5. Numerical results. Our perturbation analysis suggests that as $\rho$ increases, the mean speed of a traveling pulse decreases, and, at least for sufficiently large periods $\varepsilon$ of the weight modulation, wave propagation failure can occur. However, one of the simplifying assumptions of our analysis is that the perturbed solution is still a traveling pulse; that is, at each time $t$ there is a single bounded interval over which the solution is above threshold, which is equal to the pulse width $a$ of the homogeneous pulse in the limit $\varepsilon \rightarrow 0$. The inclusion of a periodic modulation of a monotonically decreasing weight function suggests that the assumption of a single pulse solution may break down as $\rho$ increases toward unity. In this section we explore this issue by numerically solving the full system of equations (2.1) in the case of a Heaviside nonlinearity $(f=H)$, and we show that wave propagation can persist in the presence of multiple bumps. Numerical simulations of propagating pulses are carried out using MATLAB. Initial conditions are taken to be solutions to the homogeneous problem given by (4.7) and (4.8). We then apply backward Euler to the linear terms and forward Euler with a Riemann sum to the convolution operator. Space and time discretizations are taken to be $\Delta t=0.01$ and $\Delta x=0.01$. The numerical results are stable with respect to reductions in the mesh size provided that $\Delta x \ll 2 \pi \varepsilon$. Finally, boundary points evolve freely, rather than by prescription, and the domain size is wide enough so that pulses are unaffected by boundaries.

In Figure 5 we show some examples of traveling pulse solutions in an inhomogeneous 

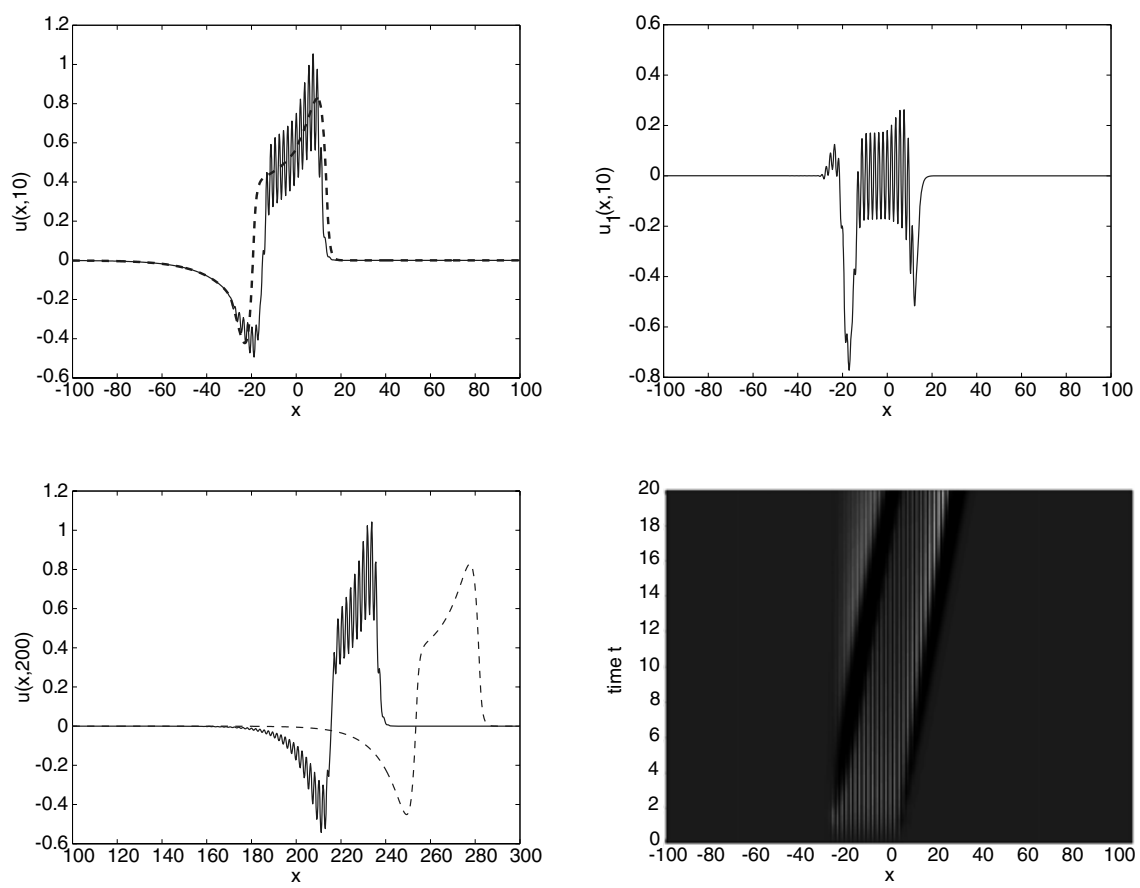

Figure 6. Top left: Comparison of the wave profiles at time $t=10$ for the homogeneous (dashed line) and the inhomogeneous (solid line) cases. Here, the parameters are $\kappa=0.2, \beta=2.0, \alpha=0.04, \varepsilon=0.3$, and $\rho=0.3$. Including periodic modulation clearly thins the pulse as we see its profile fits within that of the homogeneous medium. Top right: Subtraction of the homogeneous solution from the inhomogeneous solution at time $t=10$. We see here an approximation of $u_{1}(x, t)$, from our perturbation analysis. The dominant detail is the oscillations with period $2 \pi \varepsilon$. Bottom left: Profile comparison at $t=200$. The homogeneous solution has moved well ahead of the inhomogeneous solution due to speed difference. Bottom right: Pseudocolor plot of $u_{1}(x, t)$, obtained by subtracting the homogeneous solution from the inhomogeneous solution. The dark bands delineate the underlying homogeneous solution.

network with weight distribution given by (2.4), (2.5), and (2.6). The period of the modulation is taken to be relatively small $(\varepsilon=0.1)$. We take as initial conditions the invariant profile for the corresponding homogeneous case, obtained by solving in traveling wave coordinates for the $\varepsilon=0$ case, which gives $(U, V)$ in (4.7) and (4.8). It can be seen from Figure 5 that as the amplitude $\rho$ of the periodic modulation increases the wave slows down and narrows, which is consistent with our perturbation analysis. Moreover, the network activity develops a rippling within the interior of the pulse, as can be seen more clearly in Figure 6, where we directly compare the numerical solution of the homogeneous network with that of a corresponding inhomogeneous network. Superimposing the two wave profiles at an early time $(t=10)$ illustrates the thinning of the pulse and shows that the difference between the two wave profiles is an oscillatory component of approximately zero mean, which would correspond to $u_{1}$ in our perturbation analysis. Similarly, comparing the two wave profiles at a later time $(t=200)$ illustrates the slowing down of the pulse. As $\rho$ increases, the amplitude of the ripples also increases such that, for sufficiently large $\rho$, activity at any given time $t$ alternates between superthreshold and subthreshold domains. This is illustrated in Figure 7. A closer 

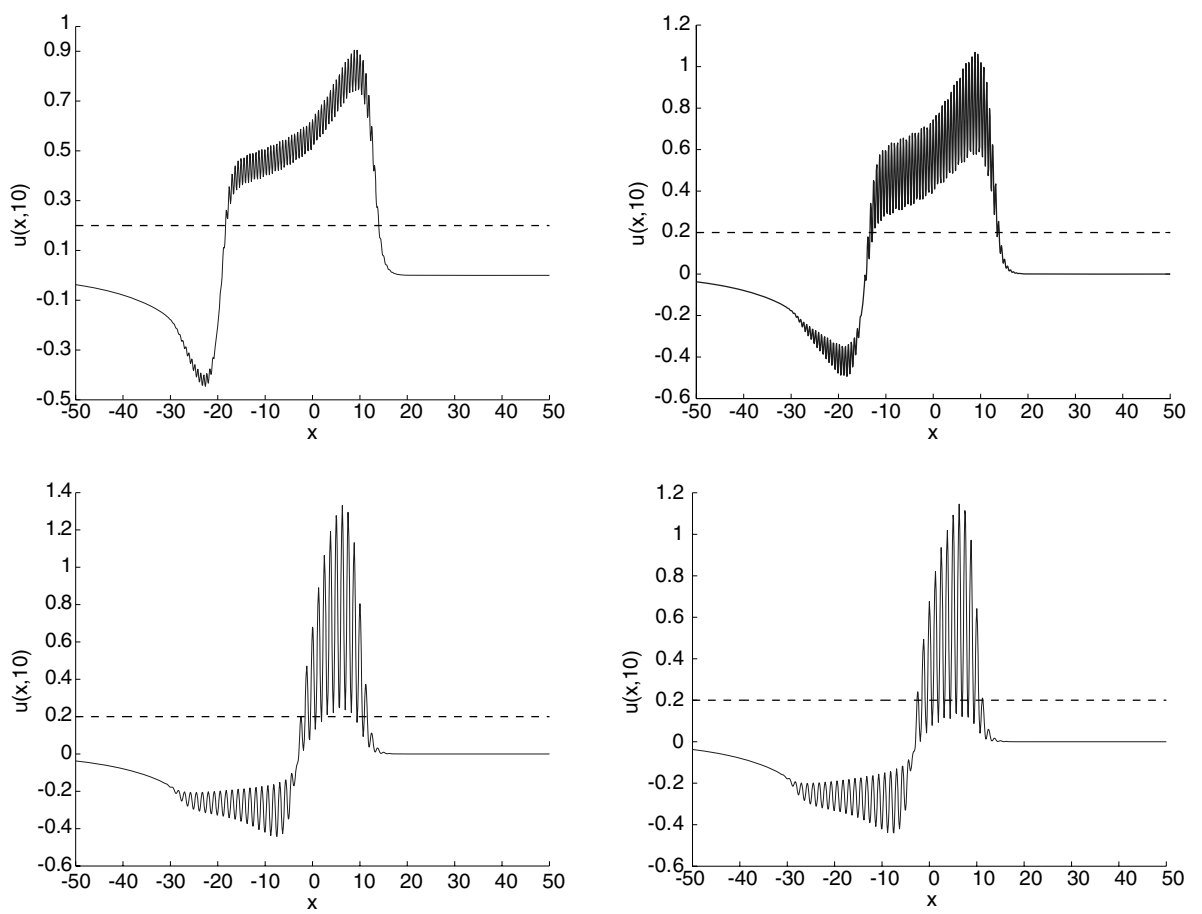

Figure 7. A collection of traveling wave profiles taken at time $t=10$ for various amplitudes $\rho$ and $\varepsilon=0.1$. Other parameters are as in Figure 5. Top left: $\rho=0.1$. Notice that rippling of the activity does not dip below threshold within the pulse interior. Top right: $\rho=0.3$. Rippling crosses below threshold at the edges of the pulse creating a couple of bumps. Bottom left: $\rho=0.7$. Rippling now generates a multiple bump solution. Bottom right: $\rho=0.8$.

look at the time evolution of the wave profile when the rippling is above threshold within the interior of the pulse shows that individual ripples are nonpropagating and transient, with new ripples appearing at the leading edge of the wave and subsequently disappearing at the trailing edge; see Figure 8. Interestingly, such behavior persists for large $\rho$ when the ripples cross below threshold within the interior of the pulse; see Figure 9. Now the pulse actually consists of multiple bumps, each of which is nonpropagating but only exists for a finite length of time. The sequence of events associated with the emergence and disappearance of these bumps generates a wave envelope that behaves very much like a single coherent traveling pulse. Hence, for sufficiently short wavelength oscillatory modulations of the weight distribution, the transient multiple bump solution can be homogenized and treated as a single traveling pulse. However, the wave speed of the multiple bump solution differs from that predicted using perturbation theory. This is shown in Figure 10, where we compare the $\bar{c}$ versus $\varepsilon$ curves obtained using perturbation theory with data obtained by directly simulating the full system (2.1). In the case of small $\rho$, a stable (single bump) traveling pulse persists for all $\varepsilon, 0 \leq \varepsilon<1$, and $\bar{c}$ is a monotonically decreasing function of $\varepsilon$. Moreover, the numerically calculated value of the average wave speed agrees quite well with the first-order perturbation analysis. On the other hand, for large values of $\rho$, such agreement no longer holds, and we find that the traveling pulse destabilizes at a critical value of $\varepsilon$ that is well below the value predicted from 

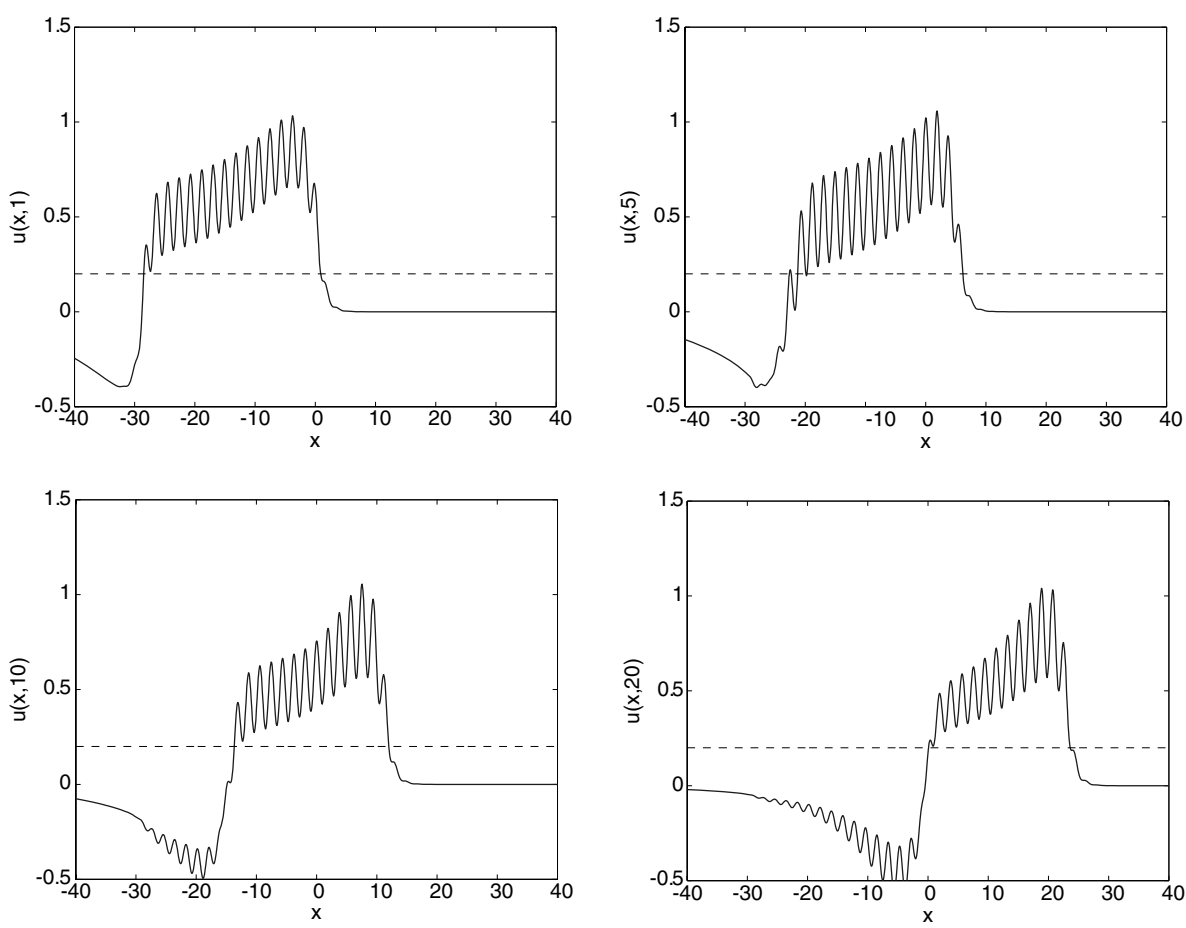

Figure 8. A series of snapshots in time of a traveling pulse for $\kappa=0.2, \beta=2.0, \alpha=0.04, \rho=0.3$, $\varepsilon=0.3$. The interior of the pulse consists of nonpropagating, transient ripples. The disappearance of ripples at one end and the emergence of new ripples at the other end generate the propagation of activity. Notice that the solitary wave profile is not invariant, reflecting the underlying inhomogeneity. Top left: $t=1$. Top right: $t=5$. Bottom left: $t=10$. Bottom right: $t=20$. Clicking on the above images displays the accompanying movie (69921_01.avi [1.7MB]).

the perturbation analysis.

In Figure 11 we compare the behavior of traveling pulses for short wavelength $(\varepsilon=0.2)$ and long wavelength $(\varepsilon=0.9)$ periodic modulation. The amplitude is taken to be relatively large, $\rho=0.8$, so that multiple bump solutions occur. We see that for long wavelength modulation, the initial pulse transitions into a nonpropagating multiple bump solution, with successive bumps disappearing sequentially and no additional bumps being created; the failure to generate new bumps means that activity cannot propagate. We can see this more clearly when examining a series of snapshots of the pulse/bump profiles in Figure 12. In conclusion, one way to understand wave propagation failure for large $\rho$ is to note that a large amplitude periodic weight modulation can generate a pinned multiple bump solution. However, in the absence of any inhibition, such a multiple bump solution is unstable [26, 37]. In the case of small $\varepsilon$, destabilization of the bumps generates new bumps at the leading edge of the bump such that activity can propagate in a coherent fashion. Increasing $\varepsilon$ prevents the creation of new bumps and propagation failure occurs.

The effect of the periodic weight modulation on a different type of solution is illustrated in Figure 13, where, motivated by a prior numerical study of multiple bumps [25], the initial condition of the network is taken to consist of three bumps, 

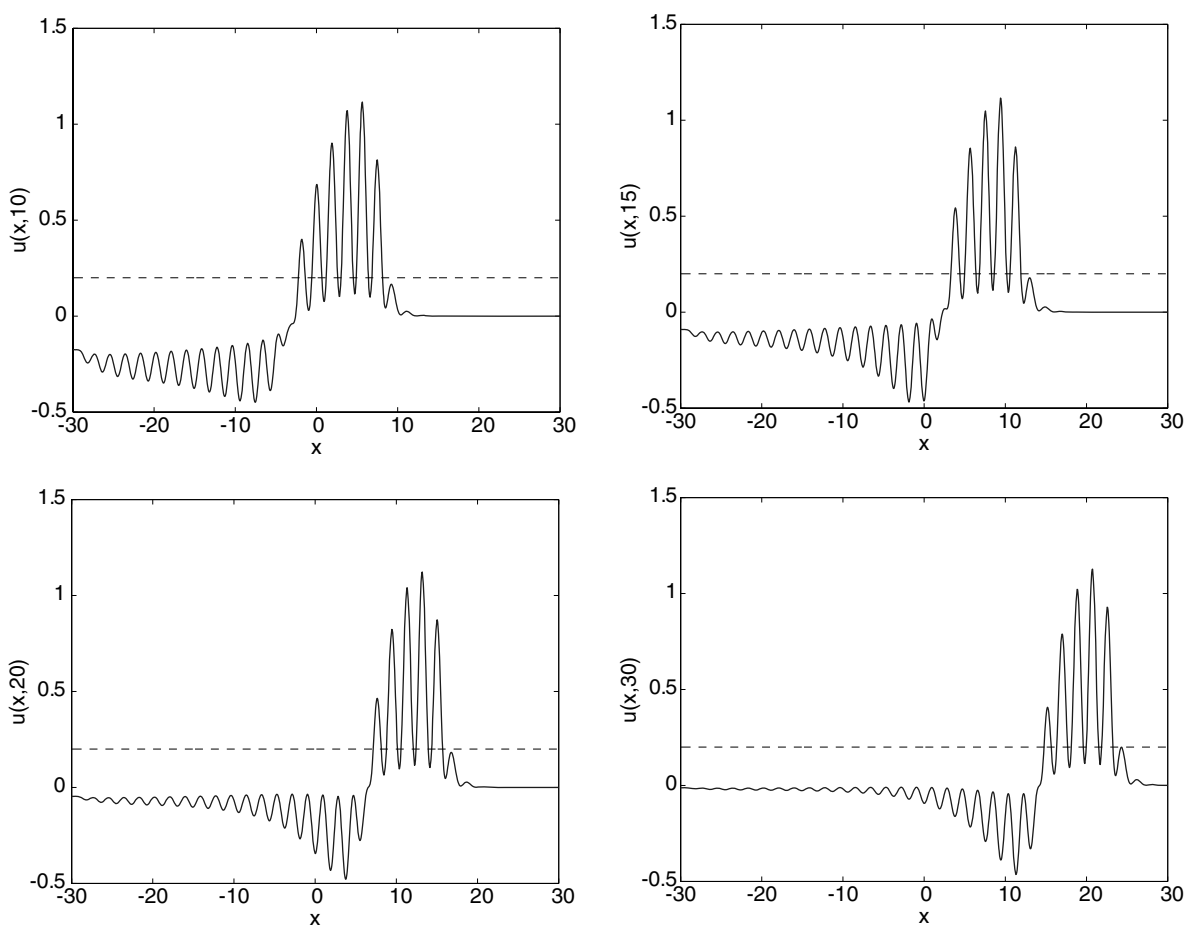

Figure 9. A series of snapshots in time of the "pulse" profile for $\kappa=0.2, \beta=2.0, \alpha=0.04, \rho=0.8$, $\varepsilon=0.3$. The solitary pulse corresponds to the envelope of a multiple bump solution, in which individual bumps are nonpropagating and transient. The disappearance of bumps at one end and the emergence of new bumps at the other end generate the propagation of activity. Notice that the solitary wave profile is not invariant, reflecting the underlying inhomogeneity. Top left: $t=10$. Top right: $t=15$. Bottom left: $t=20$. Bottom right: $t=30$. Clicking on the above images displays the accompanying movie (69921_02.avi [1.9MB]).

$$
u(x, 0)=\sum_{j=-1}^{1} \cos \left(\frac{x}{\varepsilon}\right) \exp \left(-\left(\frac{0.1(x-j \cdot 20)}{\varepsilon}\right)^{2}\right) .
$$

Each initial bump generates a pair of left and right moving fronts. In the homogeneous case, we see that collision of left and right moving waves results in a bidirectional front. That is, the region within the interior of the boundary formed by the two outermost fronts becomes superthreshold. In the inhomogeneous case, the collision of the waves is insufficient to maintain activity across this region, and one finds a pair of counterpropagating pulses.

6. Discussion. In this paper we analyzed wave propagation in an excitatory neural network treated as a periodic excitable medium. The periodicity was introduced as an inhomogeneous periodic modulation in the long-range synaptic connections and was motivated by the existence of patchy horizontal connections in the cerebral cortex. We showed that for small amplitude, short wavelength periodic modulation the main effect of the inhomogeneity is to slow down a traveling pulse, and the mean speed of the pulse can be estimated quite well using perturbation theory. In the case of large amplitude modulation, a stable traveling pulse still exists for sufficiently small $\varepsilon$, but now the pulse is the envelope of a multiple bump 


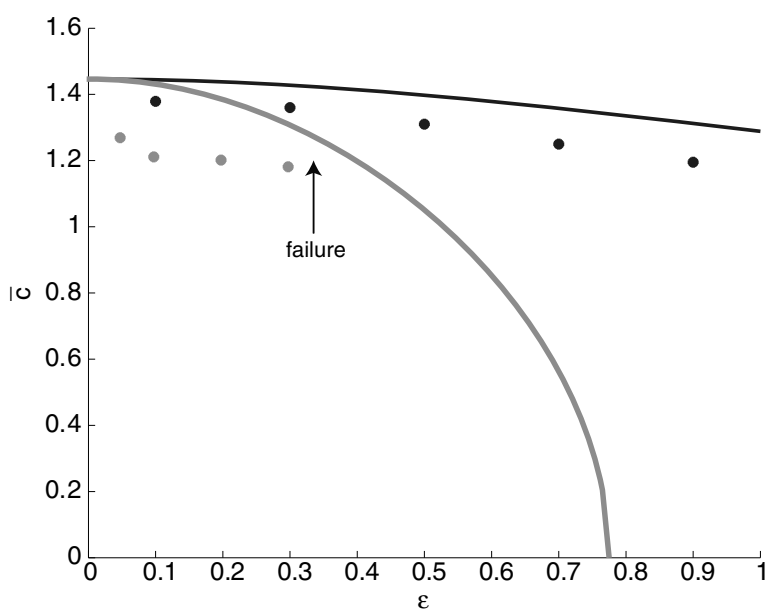

Figure 10. Comparison of perturbation theory with direct numerical simulations. Continuous curves show average wave speed $\bar{c}$ as a function of $\varepsilon$ obtained using perturbation theory. Data points are the corresponding wave speeds determined from numerically solving (2.1). In the case of low amplitude modulation $(\rho=0.3$, dark curve) a stable traveling pulse persists for all $\varepsilon, \varepsilon<1$, whereas for large amplitude modulation $(\rho=0.8$, light curve), wave propagation failure occurs as $\varepsilon$ increases.
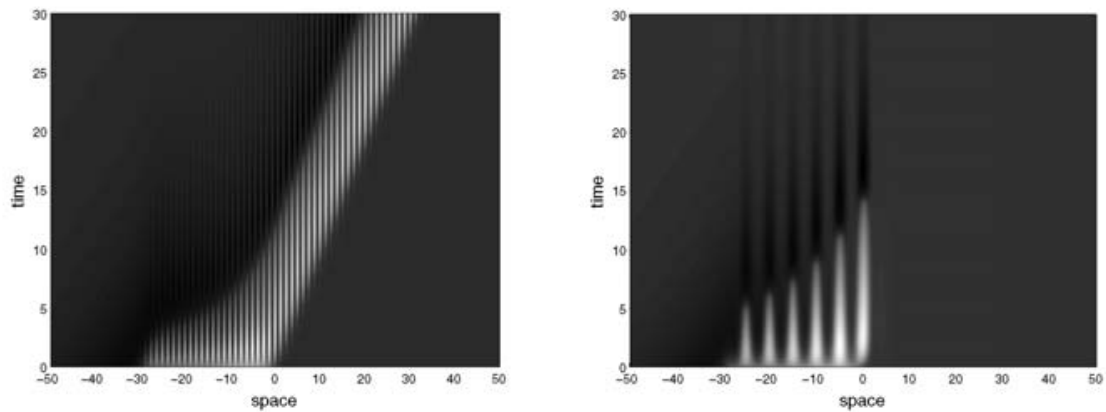

Figure 11. Comparison of traveling pulses in the case of short and long wavelength periodic modulation with $\rho=0.8$ and all other parameters as in Figure 5. Left: For short wavelength modulation $(\varepsilon=0.2)$ the traveling pulse shrinks and slows but does not annihilate. Right: For long wavelength modulation $(\varepsilon=0.9)$ wave propagation failure occurs. The initial pulse transitions into a collection of multiple equal width stationary bumps which are unstable.

solution in which individual bumps are unstable and transient. Wave propagation arises via the appearance (disappearance) of bumps at the leading (trailing) edge of the pulse. As $\varepsilon$ increases, wave propagation failure occurs due to the fact that there is insufficient activity to generate new bumps.

Although the existence of multiple bump traveling "pulses" is interesting from a dynamical systems perspective, it is less clear whether such solutions can be observed in real neural tissue. One of the biological limitations of the integrodifferential equations used in this and other studies is that, although these equations support traveling waves that have speeds consistent with neurophysiology, the pulses tend to be too wide. That is, taking the range of synaptic connections to be $1 \mathrm{~mm}$, the width of a stable pulse tends to vary between 5-30 

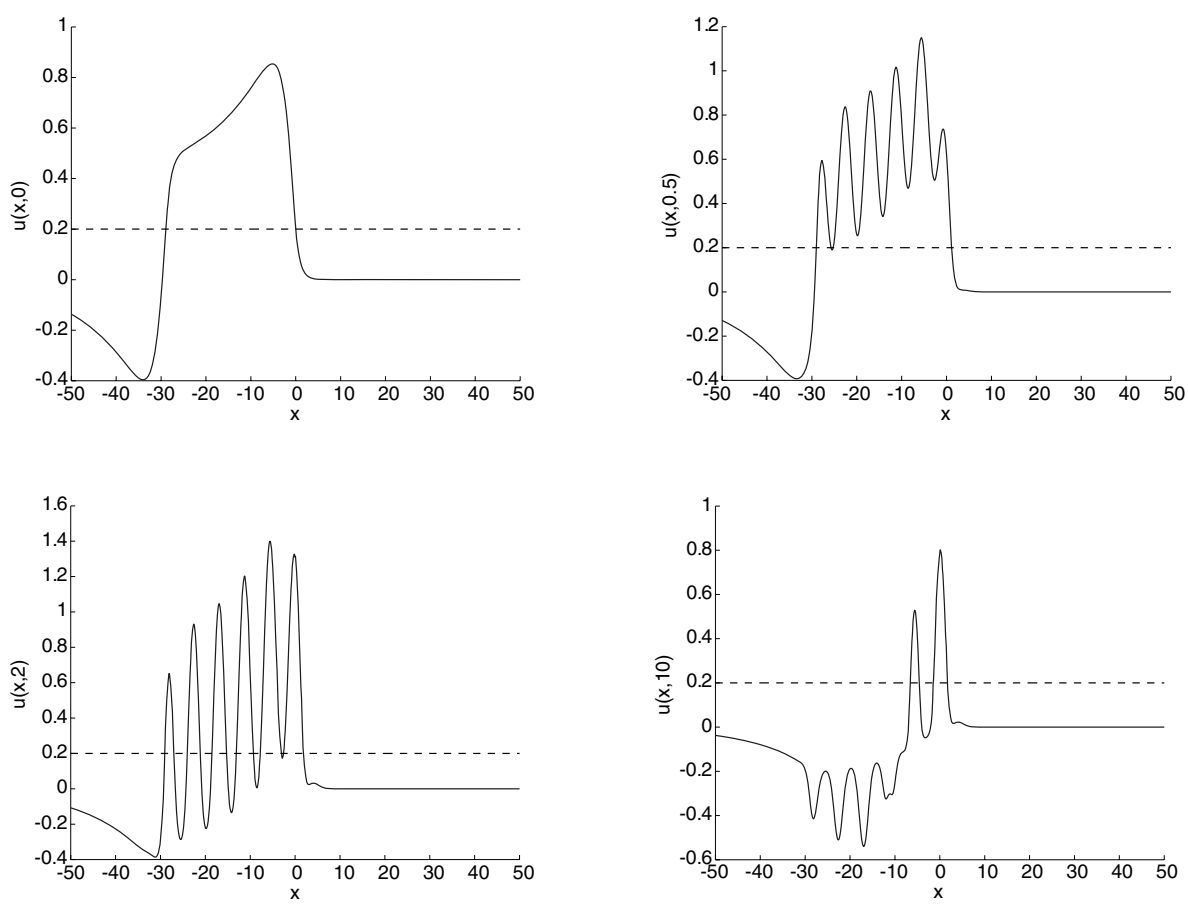

Figure 12. A series of snapshots in time of the "pulse" profile for the inhomogeneous network with $\kappa=0.2$, $\beta=2.0, \alpha=0.04, \rho=0.8, \varepsilon=0.9$. Top left: The initial wave profile, which is taken to be the invariant wave profile $U$ of the homogeneous network. Top right: Shortly after the simulation begins $(t=0.5)$, the interior of the pulse develops ripples such that the active region contains a subregion for which activity is subthreshold. Bottom left: At time $t=2$ a multiple bump profile has emerged. We can really see here how a multiple bump solution, as defined by multiple neighboring standing profiles, emerges from the pulse profile of $t=0$. Bottom right: Collapse of the pulse interior occurs due to the disappearance of the unstable bumps. Since no new bumps emerge, there is no propagating activity. Clicking on the above images displays the accompanying movie (69921_03.avi [1.1MB]).
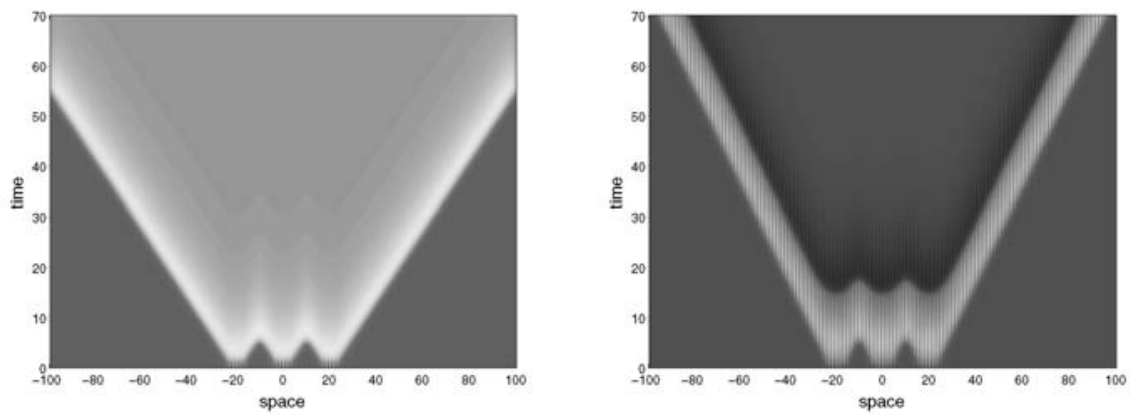

Figure 13. Left: In the case of a homogeneous network, a three bump initial condition evolves into a bidirectional front following the collision of left and right traveling waves. The parameters are $\kappa=0.2, \beta=2.0$, $\alpha=0.04$. Right: In the corresponding inhomogeneous network with $\varepsilon=0.2$ and $\rho=0.8$, the collision of left and right traveling waves results in a pair of counterpropagating pulses. Here the modulated synaptic interactions are insufficient to maintain activity in the region between the two pulses.

Copyright @ by SIAM. Unauthorized reproduction of this article is prohibited. 
$\mathrm{mm}$ (see Figure 2), whereas waves in slices tend to be only $1 \mathrm{~mm}$ wide [31]. More realistic widths and wave speeds could be generated by taking the effective range of synaptic connections to be a few hundred $\mu \mathrm{m}$, that is, by assuming that the predominant contribution to synaptic excitability is via local circuitry rather than via long-range patchy horizontal connections. However, inhomogeneities occurring at smaller spatial scales are unlikely to exhibit any periodic structure.

Irrespective of these particular issues, our analysis raises a more general point that would be interesting to pursue experimentally; namely, is it possible to detect the effects of network inhomogeneities by measuring the properties of traveling waves? Signatures of such inhomogeneities would include time-dependent rippling of the wave profile and variations in wave speed. However, such features may not be detectable given the current resolution of microelectrode recordings.

Appendix. In this appendix we present the explicit parameter-dependent expressions for the various coefficients appearing in the solution of the phase function $\Phi_{1}$, (4.28). First, the constants premultiplying the periodic functions on the right-hand side of (4.28) are as follows:

$$
\begin{aligned}
& \Xi_{ \pm}=\frac{\gamma_{ \pm}}{1+\mu_{ \pm}^{2} \varepsilon^{2}}\left[\frac{1}{2\left(1+\mu_{ \pm}\right)}+\frac{\chi}{2}\left(\frac{\mathrm{e}^{-a}-\mathrm{e}^{-\mu_{ \pm} a}}{\mu_{ \pm}-1}+\frac{\mathrm{e}^{-\mu_{ \pm} a}}{\mu_{ \pm}+1}\right)\right] \\
& \Pi_{ \pm}=\frac{\gamma_{ \pm}}{1+\mu_{ \pm}^{2} \varepsilon^{2}}\left[-\frac{\chi}{2\left(1+\mu_{ \pm}\right)}-\frac{\mathrm{e}^{-a}}{2\left(\mu_{ \pm}+1\right)}\right] \\
& \Upsilon_{ \pm}=\frac{\gamma_{ \pm}}{1+\mu_{ \pm}^{2} \varepsilon^{2}}\left[-\frac{\mu_{ \pm} \varepsilon}{2\left(1+\mu_{ \pm}\right)}-\frac{\chi \mu_{ \pm} \varepsilon}{2}\left(\frac{\mathrm{e}^{-a}-\mathrm{e}^{-\mu_{ \pm} a}}{\mu_{ \pm}-1}+\frac{\mathrm{e}^{-\mu_{ \pm} a}}{\mu_{ \pm}+1}\right)\right] \\
& \Psi_{ \pm}=\frac{\gamma_{ \pm}}{1+\mu_{ \pm}^{2} \varepsilon^{2}}\left[\frac{\chi \mu_{ \pm} \varepsilon}{2\left(1+\mu_{ \pm}\right)}+\frac{\mu_{ \pm} \varepsilon \mathrm{e}^{-a}}{2\left(1+\mu_{ \pm}\right)}\right]
\end{aligned}
$$

Second, the constant scaling factor $K$ on the left-hand side of (4.28) is determined by substituting (4.7), (4.8), and (4.14) into (3.14). Using the fact that the null-vector is zero for $\xi<-a$, we can expand the integral in terms of definite integrals of exponential products with the $\mathcal{M}_{ \pm}(\xi)$ functions

$$
\begin{aligned}
K= & {\left[\gamma_{+}\left(1-m_{-}\right)\left(1+\chi e^{-\mu_{+} a}\right)\left(1-\alpha^{-1} \beta^{-1}\left(1-m_{+}\right)^{2}\right)\right] \int_{0}^{\infty} e^{-\mu_{+} \xi} \mathcal{M}_{+}^{\prime}(\xi) \mathrm{d} \xi } \\
& +\left[\gamma_{-}\left(1-m_{-}\right)\left(1+\chi e^{-\mu_{-} a}\right)\left(1+\alpha^{-1} \beta^{-1}\left(m_{+}-1\right)\left(1-m_{-}\right)\right)\right] \int_{0}^{\infty} e^{-\mu_{-} \xi} \mathcal{M}_{+}^{\prime}(\xi) \mathrm{d} \xi \\
& -\left[\gamma_{+}\left(1-m_{+}\right)\left(1+\chi e^{-\mu_{+} a}\right)\left(1+\alpha^{-1} \beta^{-1}\left(m_{-}-1\right)\left(1-m_{+}\right)\right)\right] \int_{0}^{\infty} e^{-\mu_{+} \xi} \mathcal{M}_{-}^{\prime}(\xi) \mathrm{d} \xi \\
& -\left[\gamma_{-}\left(1-m_{+}\right)\left(1+\chi e^{-\mu_{-} a}\right)\left(1-\alpha^{-1} \beta^{-1}\left(1-m_{-}\right)^{2}\right)\right] \int_{0}^{\infty} e^{-\mu_{-} \xi} \mathcal{M}_{-}^{\prime}(\xi) \mathrm{d} \xi \\
& +\chi\left[\gamma_{+} e^{-\mu_{+} a}\left(1-m_{-}\right)\left(1-\alpha^{-1} \beta^{-1}\left(1-m_{+}\right)^{2}\right)\right] \int_{-a}^{0} e^{-\mu_{+} \xi \mathcal{M}_{+}^{\prime}(\xi) \mathrm{d} \xi} \\
& +\chi\left[\gamma_{-} e^{-\mu_{-} a}\left(1-m_{-}\right)\left(1+\alpha^{-1} \beta^{-1}\left(m_{+}-1\right)\left(1-m_{-}\right)\right)\right] \int_{-a}^{0} e^{-\mu_{-} \xi \mathcal{M}_{+}^{\prime}(\xi) \mathrm{d} \xi}
\end{aligned}
$$

Copyright $@$ by SIAM. Unauthorized reproduction of this article is prohibited. 


$$
\begin{aligned}
& -\chi\left[\gamma_{+} e^{-\mu_{+} a}\left(1-m_{+}\right)\left(1+\alpha^{-1} \beta^{-1}\left(m_{-}-1\right)\left(1-m_{+}\right)\right)\right] \int_{-a}^{0} e^{-\mu_{+} \xi} \mathcal{M}_{-}^{\prime}(\xi) \mathrm{d} \xi \\
& -\chi\left[\gamma_{-} e^{-\mu_{-} a}\left(1-m_{+}\right)\left(1-\alpha^{-1} \beta^{-1}\left(1-m_{-}\right)^{2}\right)\right] \int_{-a}^{0} e^{-\mu_{-} \xi} \mathcal{M}_{-}^{\prime}(\xi) \mathrm{d} \xi
\end{aligned}
$$

The individual integrals can be computed as follows:

$$
\begin{gathered}
\int_{0}^{\infty} e^{-\mu_{ \pm} \xi} \mathcal{M}_{ \pm}^{\prime}(\xi) \mathrm{d} \xi=\frac{e^{-a}-1}{2 c\left(m_{+}-m_{-}\right)\left(\mu_{ \pm}+1\right)^{2}}, \\
\int_{0}^{\infty} e^{-\mu_{+} \xi} \mathcal{M}_{-}^{\prime}(\xi) \mathrm{d} \xi=\frac{e^{-a}-1}{2 c\left(m_{+}-m_{-}\right)\left(\mu_{-}+1\right)\left(\mu_{+}+1\right)}, \\
\int_{0}^{\infty} e^{-\mu_{-} \xi} \mathcal{M}_{+}^{\prime}(\xi) \mathrm{d} \xi=\frac{e^{-a}-1}{2 c\left(m_{+}-m_{-}\right)\left(\mu_{+}+1\right)\left(\mu_{-}+1\right)},
\end{gathered}
$$

and

$$
\begin{gathered}
\int_{-a}^{0} e^{-\mu_{ \pm} \xi} \mathcal{M}_{ \pm}^{\prime}(\xi) \mathrm{d} \xi=\frac{1}{2 c\left(m_{+}-m_{-}\right)}\left\{\frac{a}{\left(\mu_{ \pm}-1\right)}+\frac{1-e^{\left(\mu_{ \pm}-1\right) a}}{\left(\mu_{ \pm}-1\right)^{2}}\right. \\
\left.+\frac{e^{-a}\left(e^{\left(\mu_{ \pm}+1\right) a}-1\right)}{\left(\mu_{ \pm}+1\right)^{2}}-\frac{a}{2\left(\mu_{ \pm}+1\right)}\right\} \\
\int_{-a}^{0} e^{-\mu_{+} \xi} \mathcal{M}_{-}^{\prime}(\xi) \mathrm{d} \xi= \\
\frac{1}{2 c\left(m_{+}-m_{-}\right)}\left\{\frac{1-e^{-\left(\mu_{-}-\mu_{+}\right) a}}{\left(\mu_{-}-\mu_{+}\right)\left(\mu_{-}-1\right)}-\frac{e^{\left(\mu_{+}-1\right) a}-1}{\left(\mu_{+}-1\right)\left(\mu_{-}-1\right)}\right. \\
\left.+\frac{e^{\mu_{+} a}-e^{-a}}{\left(\mu_{+}+1\right)\left(\mu_{-}+1\right)}-\frac{1-e^{-\left(\mu_{-}-\mu_{+}\right) a}}{\left(\mu_{-}+1\right)\left(\mu_{-}-\mu_{+}\right)}\right\}, \\
\int_{-a}^{0} e^{-\mu_{-} \xi \mathcal{M}_{+}^{\prime}(\xi) \mathrm{d} \xi=} \\
\frac{1}{2 c\left(m_{+}-m_{-}\right)}\left\{\frac{1-e^{-\left(\mu_{+}-\mu_{-}\right) a}}{\left(\mu_{+}-1\right)\left(\mu_{+}-\mu_{-}\right)}-\frac{e^{\left(\mu_{-}-1\right) a}}{\left(\mu_{+}-1\right)\left(\mu_{-}-1\right)}\right. \\
\left.+e^{-a} \frac{e^{\left(\mu_{-}+1\right) a}-1}{\left(\mu_{+}+1\right)\left(\mu_{-}+1\right)}-\frac{1-e^{-\left(\mu_{+}-\mu_{-}\right) a}}{\left(\mu_{+}+1\right)\left(\mu_{+}-\mu_{-}\right)}\right\} .
\end{gathered}
$$

\section{REFERENCES}

[1] S. Amari, Dynamics of pattern formation in lateral inhibition type neural fields, Biol. Cybernet., 27 (1977), pp. 77-87.

[2] W. H. Bosking, Y. Zhang, B. Schofield, And D. FitzPatrick, Orientation selectivity and the arrangement of horizontal connections in tree shrew striate cortex, J. Neurosci., 17 (1997), pp. 21122127.

[3] P. C. BRessloff, Traveling fronts and wave propagation failure in an inhomogeneous neural network, Phys. D, 155 (2001), pp. 83-100.

[4] P. C. BRessloff, Spatially periodic modulation of cortical patterns by long-range horizontal connections, Phys. D, 185 (2003), pp. 131-157.

Copyright (c) by SIAM. Unauthorized reproduction of this article is prohibited. 
[5] P. C. Bressloff and S. E. Folias, Front bifurcations in an excitatory neural network, SIAM J. Appl. Math., 65 (2004), pp. 131-151.

[6] P. C. Bressloff, Pattern formation in visual cortex, in Methods and Models in Neurophysics, C. C. Chow, B. Gutkin, D. Hansel, C. Meunier, and J. Dalibard, eds., Les Houches Lectures in Neurophysics, Springer-Verlag, Berlin, 2004, pp. 477-574.

[7] R. D. Chervin, P. A. Pierce, And B. W. Connors, Periodicity and directionality in the propagation of excitation in neural network model, J. Neurophysiol., 60 (1988), pp. 1695-1713.

[8] B. W. Connors And Y. Amitai, Generation of epileptiform discharge by local circuits of neocortex, in Epilepsy: Models, Mechanisms and Concepts, P. A. Schwartkroin, ed., Cambridge University Press, Cambridge, UK, 1993, pp. 388-423.

[9] S. Coombes And M. R. Owen, Bumps, breathers, and waves in a neural network with spike frequency adaptation, Phys. Rev. Lett., 94 (2005), 148102.

[10] S. Coombes And M. R. Owen, Evans functions for integral neural field equations with Heaviside firing rate function, SIAM J. Appl. Dyn. Syst., 3 (2004), pp. 574-600.

[11] S. Coombes, Waves, bumps, and patterns in neural field theories, Biol. Cybernet., 93 (2005), pp. 91-108.

[12] G. B. Ermentrout And J. B. MCLeOd, Existence and uniqueness of travelling waves for a neural network, Proc. Roy. Soc. Edinburgh Sect. A, 123 (1993), pp. 461-478.

[13] G. B. ERmentrout And D. Kleinfeld, Traveling electrical waves in cortex: Insights from phase dynamics and speculation on a computational role, Neuron, 29 (2001), pp. 33-44.

[14] S. E. Folias AND P. C. BREssloff, Stimulus-locked traveling waves and breathers in an excitatory neural network, SIAM J. Appl. Math., 65 (2005), pp. 2067-2092.

[15] D. Golomb and Y. Amitai, Propagating neuronal discharges in neocortical slices: Computational and experimental study, J. Neurophysiol., 78 (1997), pp. 1199-1211.

[16] J. Guckenheimer And P. J. Holmes, Nonlinear Oscillations, Dynamical Systems, and Bifurcations of Vector Fields, Springer-Verlag, New York, 1983.

[17] B. S. Gutkin, G. B. Ermentrout, and J. O'Sullivan, Layer 3 patchy recurrent excitatory connections may determine the spatial organization of sustained activity in the primate prefrontal cortex, Neurocomputing, 32-33 (2000), pp. 391-400.

[18] X. Huang, W. C. Troy, Q. Yang, H. Ma, C. Laing, S. Schiff, and J. Y. Wu, Spiral waves in disinhibited mammalian cortex, J. Neurosci., 24 (2004), pp. 9897-9902.

[19] M. A. P. Idiart AND L. F. ABbott, Propagation of excitation in neural network models, Network, 4 (1993), pp. 285-294.

[20] J. P. KEEnER, Propagation of waves in an excitable medium with discrete release sites, SIAM J. Appl. Math., 61 (2000), pp. 317-334.

[21] J. P. KeEner, Homogenization and propagation in the bistable equation, Phys. D, 136 (2000), pp. 1-17.

[22] D. Kleinfeld, K. R. Delaney, M. S. Fee, J. A. Flores, D. W. Tank, and A. Galperin, Dynamics of propagating waves in the olfactory network of a terrestrial mollusk: An electrical and optical study, J. Neurophysiol., 72 (1994), pp. 1402-1419.

[23] Y. W. Lam, L. B. Cohen, M. Wachowiak, and M. R. Zochowski, Odors elicit three different oscillations in the turtle olfactory bulb, J. Neurosci., 20 (2000), pp. 749-762.

[24] J. W. LANCE, Current concepts of migraine pathogenesis, Neurology, 43 (1993), pp. 11-15.

[25] C. R. Laing, W. C. Troy, B. Gutkin, and G. B. Ermentrout, Multiple bumps in a neuronal model of working memory, SIAM J. Appl. Math., 63 (2002), pp. 62-97.

[26] C. R. LAING AND W. C. TROy, Two-bump solutions of Amari-type models of neuronal pattern formation, Phys. D, 178 (2003), pp. 190-218.

[27] J. B. Levitt, D. A. Lewis, T. Yoshioka, And J. S. Lund, Topography of pyramidal neuron intrinsic connections in macaque prefrontal cortex, J. Comp. Neurol., 338 (1993), pp. 360-376.

[28] R. Malach, Y. Amir, M. Harel, And A. Grinvald, Relationship between intrinsic connections and functional architecture revealed by optical imaging and in vivo targeted bicytin injections in primate striate cortex, Proc. Natl. Acad. Sci. USA, 90 (1993), pp. 10469-10473.

[29] D. S. Melchitzky, S. R. Sesack, M. L. Pucak, and D. A. Lewis, Synaptic targets of pyramidal neurons providing intrinsic horizontal connections in monkey prefrontal cortex, J. Comp. Neurol., 390 (1998), pp. 211-224.

Copyright (C) by SIAM. Unauthorized reproduction of this article is prohibited. 
[30] M. A. L. Nicolelis, L. A. Baccala, R. C. S. Lin, And J. K. Chapin, Sensorimotor encoding by synchronous neural ensemble activity at multiple levels of the somatosensory system, Science, 268 (1995), pp. 1353-1358.

[31] D. J. Pinto And G. B. Ermentrout, Spatially structured activity in synaptically coupled neuronal networks: I. Traveling fronts and pulses, SIAM J. Appl. Math., 62 (2001), pp. 206-225.

[32] D. J. Pinto, S. L. Patrick, W. C. Huang, And B. W. Connors, Initiation, propagation, and termination of epileptiform activity in rodent neocortex in vitro involve distinct mechanisms, J. Neurosci., 25 (2005), pp. 8131-8140.

[33] D. J. Pinto, R. K. Jackson, and C. E. Wayne, Existence and stability of traveling pulses in a continuous neuronal network, SIAM J. Appl. Dyn. Syst., 4 (2005), pp. 954-984.

[34] J. C. Prechtl, L. B. Cohen, B. Pasaram, P. P. Mitra, and D. Kleinfeld, Visual stimuli induce waves of electrical activity in turtle cortex, Proc. Natl. Acad. Sci. USA, 94 (1997), pp. 7621-7626.

[35] K. A. Richardson, S. J. Schiff, And B. J. Gluckman, Control of traveling waves in the mammalian cortex, Phys. Rev. Lett., 94 (2005), 028103.

[36] P. R. Roelfsema, A. K. Engel, P. Konig, And W. Singer, Visuomotor integration is associated with zero time-lag synchronization among cortical areas, Nature, 385 (1997), pp. 157-161.

[37] J. E. Rubin AND W. C. TROY, Sustained spatial patterns of activity in neuronal populations without recurrent excitation, SIAM J. Appl. Math., 64 (2004), pp. 1609-1635.

[38] W. C. Troy and V. Shusterman, Patterns and features of families of traveling waves in large-scale neuronal networks, SIAM J. Appl. Dyn. Syst., 6 (2007), pp. 263-292.

[39] H. R. Wilson And J. D. Cowan, A mathematical theory of functional dynamics of cortical and thalamic nervous tissue, Kybernetik, 13 (1973), pp. 55-80.

[40] J.-Y. WU, L. GUAN, AND Y. Tsau, Propagating activation during oscillations and evoked responses in neocortical slices, J. Neurosci., 19 (1999), pp. 5005-5015.

[41] T. Yoshioka, G. G. Blasdell, J. B. Levitt, and J. S. Lund, Relation between patterns of intrinsic lateral connectivity, ocular dominance, and cytochrome oxidase-reactive regions in macaque monkey striate cortex, Cerebral Cortex, 6 (1996), pp. 297-310.

[42] L. Zhang, On stability of traveling wave solutions in synaptically coupled neuronal networks, Differential Integral Equations, 16 (2003), pp. 513-536.

[43] L. ZHANG, Existence, uniqueness, and exponential stability of traveling wave solutions of some integral differential equations arising from neuronal networks, J. Differential Equations, 197 (2004), pp. 162196.

Copyright (C) by SIAM. Unauthorized reproduction of this article is prohibited. 